\title{
ON LIE ALGEBRAS OF VECTOR FIELDS
}

\author{
BY
}

\author{
AKIRA KORIYAMA, YOSHIAKI MAEDA AND HIDEKI OMORI
}

\begin{abstract}
This paper has two purposes. The first is a generalization of the theorem of Pursell-Shanks [10]. Our generalization goes by assuming the existence of a nontrivial core of a Lie algebra. However, it seems to be a necessary condition for the theorems of Pursell-Shanks type.

The second is the classification of cores under the assumption that the core itself is infinitesimally transitive at every point. As naturally expected, we have the nonelliptic, primitive infinite-dimensional Lie algebras.
\end{abstract}

0 . Introduction. Let $M$ and $N$ be connected $C^{\infty}$ manifolds and $\mathfrak{X}(M)$ (resp. $\mathfrak{X}(N)$ ) the Lie algebra of all $C^{\infty}$ vector fields with compact support on $M$ (resp. $N)$. A well-known theorem of Pursell-Shanks [10] may be stated as follows:

THEOREM. There exists a Lie algebra isomorphism $\Phi$ of $\mathfrak{X}(M)$ onto $\mathfrak{X}(N)$ if and only if there exists a $C^{\infty}$ diffeomorphism $\varphi$ of $M$ onto $N$ such that $\Phi=d \varphi$.

The above result still holds for Lie algebras of all infinitesimal automorphisms of several geometric structures on $M$ and $N$. Indeed, Omori $[9,8 \mathrm{X}]$ proved the corresponding result in case of volume structures, symplectic structures, contact structures and fibering structures with compact fibers, and Koriyama [4] showed this is still true for submanifolds regarding a submanifold $M^{\prime}$ as a geometric structure on $M$. Recently, Amemiya [2] gave a generalization of the theorem of Pursell-Shanks in the case that the Lie algebra $g$ is a module over $C^{\infty}$ functions on $M$ and there is no point on which every $u \in \mathfrak{g}$ vanishes.

Our first purpose of this paper is to give a unified proof for the above results.

Now, we consider a Lie algebra $g$ (resp. ' $g$ ) of $C^{\infty}$ vector fields on a connected, complete $C^{\infty}$ riemannian manifold $M$ (resp. $N$ ) with the following conditions:

$(C, 1)$ Every $u \in g$ is complete (integrable).

$(C, 2) \operatorname{Ad}(\exp u) \mathfrak{g}=\mathfrak{g}$ for every $u \in \mathfrak{g}$.

$(C, 3) g$ is a locally closed LAS.

$(C, 4) g$ has nontrivial core.

Received by the editors September 4, 1975.

AMS (MOS) subject classifications (1970). Primary 58A30, 58B99.

Key words and phrases. Cores of Lie algebras, multivalued primitive structures.

(4) American Mathematical Sociely 1977 
$(C, 5) \mathrm{g}$ is infinitesimally transitive at every point.

Our first main theorem is stated as follows:

TheOREM A. Let $\mathfrak{g},{ }^{\prime} \mathfrak{g}$ be Lie algebras of $C^{\infty}$ vector fields with $(\mathrm{C}, 1)-(\mathrm{C}, 5)$. Then there exists an algebraic isomorphism $\Phi: \mathfrak{g} \rightarrow$ 'g which sends a nontrivial subcore to a subcore if and only if there exists a $C^{\infty}$ diffeomorphism $\varphi: M \rightarrow N$ such that $\Phi=d \varphi$.

Now, we want to explain conditions $(C, 1)-(C, 5)$.

Condition $(\mathrm{C}, 1)$ seems to be natural from the standpoint of the theory of Lie groups. If $M$ is closed, every vector field is complete. However, if $M$ is not compact, we have to note the following: Let $e$ be the set all complete $C^{\infty}$ vector fields on $M$. Then, in general, $\mathcal{C}$ is neither a linear space nor a closed subset in the $C^{\infty}$ topology. Moreover $C$ is not closed under the Lie bracket product. Roughly speaking, Lie algebras with $(\mathrm{C}, 1)$ are obtained through various compactifications of $M$.

If $g$ has the property $(C, 1)$, then for any $u \in g$ there is a one parameter group exp $t u$ generated by $u$. This is a one parameter family of $C^{\infty}$ diffeomorphisms of $M$ onto itself. For any diffeomorphism $\varphi$, we define $\operatorname{Ad}(\varphi) v$ by $(\operatorname{Ad}(\varphi) v)(p)=d \varphi v\left(\varphi^{-1}(p)\right)$. Thus, condition $(C, 2)$ seems also natural from the standpoint of the theory of Lie groups. There are, however, a lot of Lie algebras without this condition. For instance, set $g=\{f(x) \partial / \partial x\}$ on a line $\mathbf{R}$, where $f$ is a $C^{\infty}$ function with compact support, constant on a fixed neighborhood of 0 . Then, $g$ satisfies $(C, 1)$ but not $(C, 2)$. (Indeed, $g$ satisfies $(C, 1)-(C, 5)$ except $(C, 2)$.) However, condition $(C, 2)$ does not give a strong restriction for Lie algebras. It is easy to be satisfied as we can see in $\$ 2$.

Let $g$ be a Lie algebra of $C^{\infty}$ vector fields on $M . g$ is said to be a locally closed $L A S$, if the following conditions are satisfied:

(LC) Let $\left\{u_{n}\right\}$ be a sequence in $g$ such that $\operatorname{supp} u_{n}$ are contained in a compact set $K$ for all $n$. Then $\lim _{n \rightarrow \infty} u_{n} \in \mathfrak{g}$ whenever it exists in the $C^{\infty}$ topology.

(LAS) Let $u$ be a $C^{\infty}$ vector field on $M$ with a compact support. Then $u \in \mathfrak{g}$ if and only if, for any $p \in M$ and for any integer $k \geqslant 0$, there is $v_{p}^{k} \in g$ such that $j_{p}^{k} u=j_{p}^{k} v_{p}^{k}$, where $j_{p}^{k}$ is the $k$ th jet at $p$.

Roughly speaking, the condition (LAS) means that $g$ is defined by a linear differential operator. However, this condition is not used so strictly in the proof of Theorem A. All results except the proof of Theorem B stated below hold without condition $(\mathrm{C}, 3)$ but with the following much weaker condition:

$(\mathrm{IC}, 2)$ For any $v \in g$ with a compact support,

$$
\int_{a}^{b} \operatorname{Ad}(\exp t u) v d t \in \mathfrak{g} \quad \text { for any } u \in g, \quad-\infty<a \leqslant b<\infty .
$$

(Cf. Lemma 1.2 and the remark there.) 
Condition $(\mathrm{C}, 3)$ plays an important role in the proof of the next main theorem of this paper. The Lie algebra $\mathfrak{X}(M)$ of all $C^{\infty}$ vector fields with compact support is an example of Lie algebras with $(C, 3)$.

Condition $(\mathrm{C}, 5)$ means, of course, that $\operatorname{dim}\{u(p) ; u \in g\}=\operatorname{dim} M$ at every $p \in M$. However, this condition is rather technical. Many results in this paper hold without this condition. So the results which are proved by using $(C, 5)$ will be stated as corollaries in this paper.

Now, we have to explain condition $(C, 4)$. Though the precise definition of the core of $g$ is given in $\S 1$, the most essential property of the core $\mathrm{Q}(g)$ is the following:

(P) Let $u \in \mathcal{C}(g)$. For any positive $\varepsilon, u$ can be written as a finite sum $u=\Sigma_{\alpha} u_{\alpha}$ such that $u_{\alpha} \in \mathcal{C}(\mathrm{g})$ and $\operatorname{diam}\left(\operatorname{supp} u_{\alpha}\right)<\varepsilon$, where diam(supp) means the diameter of the support.

Indeed, $\mathcal{Q}(g)$ is defined as a maximal subspace of $g$ with the above property (P) (cf. §1).

As a matter of course, $(\mathrm{P})$ is one of the properties of a subcore. The existence of a nontrivial core is essential in this paper and indeed gives the strongest restriction to the Lie algebras. Many important Lie algebras such as Lie algebras of real analytic vector fields have the trivial core. Moreover, by condition $(\mathbf{C}, 4)$, we have to exclude the Lie algebras of infinitesimal automorphisms of elliptic $G$-structures. Cores will be classified under the additional condition that the core itself is infinitesimally transitive at every point. Indeed, this is the second purpose of this paper.

THEOREM B. Let $\mathrm{G}(\mathrm{g})$ be the core of a Lie algebra with $(\mathrm{C}, 1)-(\mathrm{C}, 5)$. Assume furthermore that $\mathrm{Q}(\mathrm{g})$ is infinitesimally transitive at every point. Then $\mathrm{Q}(\mathrm{g})$ is one of the following Lie algebras: $\mathfrak{X}(M), \mathfrak{X}_{\omega}(M), \mathfrak{X}_{d V, c}(M), \mathfrak{X}_{\Omega, c}(M)$.

Though the precise definition of the above Lie algebras will be given in $\S \S 8$ and 9, we want to remark the following:

(i) $\mathfrak{X}(M)$ is the Lie algebra of all $C^{\infty}$ vector fields with compact support.

If $M$ is simply connected, then

(ii) $\mathfrak{X}_{\omega}(M)$ is the Lie algebra of all $C^{\infty}$ contact transformations with compact support.

(iii) $\mathfrak{X}_{d V, c}(M)=\{u \in \mathfrak{X}(M)$; $d V\lrcorner u=d \gamma$, supp $\gamma$ is compact $\}$.

(iv) $\mathfrak{X}_{\Omega, c}(M)=\{u \in \mathfrak{X}(M) ; \Omega\lrcorner u=d \gamma$, supp $\gamma$ is compact $\}$, where $d V, \Omega$ are a $C^{\infty}$ volume form, a $C^{\infty}$ symplectic form respectively, and $\lrcorner$ means the inner product.

If $M$ is not simply connected, then $\omega, d V, \Omega$ are multivalued forms on $M$ and the definitions of cores are a little more complicated. (For the precise definitions, see the first paragraph of $\$ 8$ and Lemma 8.4 and $\S 9$, (B).) The classification is based on the corollary of Lemma 8.2.

Now, the proof of Theorem A goes as follows: 
(1) First of all, a characterization of maximal ideals of a subcore $\mathfrak{h}$ is given. Then it can be seen that there is a one-to-one correspondence between maximal ideals $g$ such that $g D[\mathfrak{h}, \mathfrak{h}]$ and points of the manifold.

(2) Secondly, the given Lie algebra isomorphism $\Phi$ sends maximal ideals of $\mathfrak{h}$ onto those of $\mathfrak{h}=\Phi(\mathfrak{h})$, hence $\Phi$ induces a one-to-one correspondence $\varphi$ of $M$ onto $N$. This $\varphi$ turns out to be a homeomorphism.

(3) Thirdly, to prove that $\varphi$ is a diffeomorphism, we use condition $(C, 5)$ and the formula $\Phi(\operatorname{Ad}(\exp t u) v)=\operatorname{Ad}(\exp t \Phi(u)) \Phi(v)$, which will be established in 85 .

If $g$ is without $(C, 5)$, then we have only the differentiability of $\varphi$ on each $g$ orbit, where $\mathrm{g}$-orbit is an orbit of the group \& generated by $\{\exp t u ; u \in \mathfrak{g}\}$. Every g-orbit is an immersed submanifold of $M$ as we will show in \$2, but this fact has been obtained originally by Sussmann [13]. Even in such intransitive Lie algebra $\mathfrak{g}$, if $\mathfrak{g}$ contains enough information about transversal directions of orbits, the differentiability of $\varphi$ may be concluded by such information. In fact, if $g$ is a module over $C^{\infty}$ functions, the transitivity condition $(C, 5)$ can be easily replaced by the condition that there is no point on which every $u \in g$ vanishes (cf. §6). Moreover, in several concrete examples related to Hamiltonian systems, condition $(C, 5)$ can be replaced by some other weaker conditions. These examples, however, will be discussed elsewhere.

As for the Lie algebras treated in $[9, \S \mathrm{X}]$, the core is characterized algebraically by $g$. Therefore in such Lie algebras, any isomorphism sends the core to the core. Indeed, if $g$ is the Lie algebra of all infinitesimal contact transformations with compact support, the core of $g$ is $g$ itself (cf. [9, p. 98]). If $g$ is the Lie algebra of infinitesimal automorphisms which leave compact fibers invariant, then the core of $g$ is given by the intersection of all proper maximal ideals (cf. [9, p. 138]). If $g$ is the Lie algebra of infinitesimal automorphisms of a symplectic or a volume structure, then the core of $g$ is given by the derived algebra $[g, g]$ under the additional condition that $M$ is closed, where the derived algebra means the finite sum of elements $[u, v], u, v$ $\in$ g. However, Omori's proof in $[9, \S \mathrm{X}]$ contains a gap. Lemma 10.4.1 in [9] holds only for the case $M$ is closed, and Lemma 10.3 .2 is proved by using the above lemma implicitly. So the precise proof will be given in $\$ 6$ in this paper.

The proof of Theorem B is, of course, based on the classification of the primitive, complete Lie algebras, obtained by Singer and Sternberg [12], Morimoto and Tanaka [7], Shnider [11] and Guillemin [3]. The proof goes as follows:

(1) Let $\mathbb{E}$ be the core of given Lie algebra g. $\mathbb{C}$ is infinitesimally transitive at every point by the hypothesis of the theorem. First of all, we will show that the isotropy subalgebra $\mho_{p}$ of ${ }^{c}$ at $p \in M$ is a maximal subalgebra (cf. §3). We take the Taylor expansions of all $u \in \mathbb{C}$ at a point $p \in M$ and take the closure of it in the linear topology of the formal power series. Then, we get 
one of the 14 Lie algebras in the classification table of complete primitive Lie algebras.

(2) Secondly, using the transitivity of $\Subset$, we make a $G$-structure on $M$ so that the group $\mathcal{C}$ generated by $\{\exp t u ; u \in \mathbb{E}\}$ may become an auotmorphism group.

(3) Thirdly, by condition $(C, 4)$ we exclude the case that $G$ is elliptic. Thus, only the 6 cases remain.

(4) Fourthly, by case-by-case method combined with condition $(C, 3)$, we fix the core.

Step (4) is the only reason why we restrict ourselves to transitive cores instead of treating the restriction of $\mathbb{E}$ onto $\mathbb{E}$-orbit. Though many lemmas in the proof of Theorem B hold for the Lie algebra $\mathbb{E}$ restricted to a $\mathbb{E}$-orbit $\theta_{p}$, the local closedness of $\mathrm{E} \mid \theta_{p}$ is not guaranteed by that of $\mathrm{g}$.

1. Definition of the core, subcores and several properties. Let $M$ be a connected, complete $C^{\infty}$ riemannian manifold and $g$ a Lie algebra of $C^{\infty}$ vector fields on $M$ with conditions $(\mathrm{C}, 1)-(\mathrm{C}, 3)$. For any subspace $\mathfrak{h}$ of $g$, we denote by $\Sigma_{0}(\mathfrak{h})$ the subset of all common zeros of $\mathfrak{h}$. Let $\delta$ be the set of all subspaces $\mathfrak{h}$ of $\mathfrak{g}$ with the following property:

(P) For any $u \in \mathfrak{h}$, there is a compact set $K_{u}$ such that $K_{u} \cap \Sigma_{0}(\mathfrak{h})=\varnothing$ and, for any positive $\varepsilon, u$ can be written as a finite sum $u=\Sigma_{\alpha} u_{\alpha}$ such that $u_{\alpha} \in \mathfrak{h}, \operatorname{supp} u_{\alpha} \subset K_{u}$ and diam $\left(\operatorname{supp} u_{\alpha}\right)<\varepsilon$.

The above property means especially that every $u \in \mathfrak{h}$ is identically zero in a neighborhood of $\Sigma_{0}(\mathfrak{h})$.

LEMMA 1.1. If $\mathfrak{h}_{1}, \mathfrak{h}_{2} \in \delta$, then $\mathfrak{h}_{1}+\mathfrak{h}_{2} \in \delta$.

Proof. Note that $\Sigma_{0}\left(\mathfrak{h}_{1}+\mathfrak{h}_{2}\right)=\Sigma_{0}\left(\mathfrak{h}_{1}\right) \cap \Sigma_{0}\left(\mathfrak{h}_{2}\right)$. For any $u+v \in \mathfrak{h}_{1}$ $+\mathfrak{h}_{2}$, we set $K_{u+v}=K_{u} \cup K_{v}$. Then, we have $K_{u+v} \cap \Sigma_{0}\left(\mathfrak{h}_{1}+\mathfrak{h}_{2}\right)=\varnothing$. Other properties can be easily checked.

Since $\delta$ is a partially ordered set, we see by the above lemma that there is a unique maximal element $C(g)$, which will be called the core of $\mathrm{g}$.

LEMMA 1.2. Let $v$ be an element of $g$ with a compact support. Then, $\int_{a}^{b} \operatorname{Ad}(\exp t u) v d t \in g$ for any $u \in g$ and for any $a, b$ such that $-\infty<a \leqslant b$ $<\infty$ (i.e., g satsifies $(\mathrm{IC}, 2)$ ).

Proof. $K=\cup_{t \in[a, b]}$ supp Ad (exptu)v is a compact set, hence so is supp $\int_{a}^{b} \operatorname{Ad}(\exp t u) v d t$. Since the integration is a limit of finite sum, we have $\int_{a}^{b} \operatorname{Ad}(\exp t u) v d t \in g$.

REMARK. Once this property (IC,2) is established, we do not use the local closedness any more until $\S 8$.

LemMa 1.3. Suppose $u \in g$ does not vanish at $p \in M$. Then there is a relatively compact open neighborhood $V(p)$ of $p$ satisfying the following: For any 
$v \in \mathfrak{g}$ with $\operatorname{supp} v \subset V(p)$, there is $I(v) \in \mathfrak{g}$ such that $[u, I(v)] \equiv v$ on $V(p)$.

Proof. There is an open coordinate neighborhood $U(p)$ of $p$ with a coordinat. system $x_{1}, \ldots, x_{n}$ such that $u \equiv \partial / \partial x_{1}$ on $U(p)$. We choose a sufficiently small cubic neighborhood $V(p)=(-a, a) \times \cdots \times(-a, a)$ such that $(-3 a, 3 a) \times(-a, a) \times \cdots \times(-a, a)$ is still contained in $U(p)$. Now, we set $I(v)=\int_{0}^{2 a} \operatorname{Ad}(\exp t u) v d t$. By the above lemma, we see that $I(v) \in g$. On the other hand, for any $\left(x_{1}, \ldots, x_{n}\right) \in V(p)$,

$$
(\operatorname{Ad}(\exp t u) v)\left(x_{1}, \ldots, x_{n}\right)=v\left(x_{1}-t, x_{2}, \ldots, x_{n}\right) .
$$

Thus, we have the following for any $v \in g$ with supp $v \subset V(p)$ :

$$
I(v)\left(x_{1}, \ldots, x_{n}\right)=\int_{x_{1}-2 a}^{x_{1}} v\left(t, x_{2}, \ldots, x_{n}\right) d t=\int_{-a}^{x_{1}} v\left(t, x_{2}, \ldots, x_{n}\right) d t .
$$

Hence, it is clear that $[u, I(v)] \equiv v$ on $V(p)$.

Now, let $\mathcal{C}(g)$ be the core of $g$. By $[\mathcal{C}(g), g]$ we denote the space of the finite sum of elements $[u, v], u \in \mathcal{C}(\mathrm{g}), v \in \mathrm{g}$.

Lemma 1.4. $\Sigma_{0}([\mathcal{C}(\mathfrak{g}), \mathrm{g}])=\Sigma_{0}(\mathcal{C}(\mathfrak{g}))$.

Proof. Since supp $u \cap \Sigma_{0}(\mathcal{C}(g))=\varnothing$ for any $u \in \mathcal{C}(g)$ (cf. the property (P)), we have $\Sigma_{0}([\mathcal{C}(g), g]) \supset \Sigma_{0}(\mathcal{C}(g))$.

Now, for any point $p \in M-\Sigma_{0}(\mathcal{C}(g))$ there is $u \in \mathcal{C}(g)$ such that $u(p)$ $\neq 0$. Hence by the above lemma there is a relatively compact neighborhood $V(p)$ such that if $v \in g$ has the property supp $v \subset V(p)$, then $[u, I(v)] \equiv v$ on $V(p)$. On the other hand, the core has the property $(\mathrm{P})$. Therefore, there is $w \in \mathcal{C}(g)$ such that $\operatorname{supp} w \subset V(p)$ and $w(p) \neq 0$. Thus, we have $[\mathcal{C}(\mathbb{g}), g](p)$ $\neq\{0\}$ where $p \in M-\Sigma_{0}(\mathcal{C}(\mathfrak{g}))$.

The following are the properties of the core $\mathcal{Q}(g)$ of $g$ which will be used in this paper:

Proposition 1.1. Let $\mathcal{C}(\mathrm{g})$ be the core of $\mathrm{g}$ with $(\mathrm{C}, 1)-(\mathrm{C}, 3)$.

(i) $\mathcal{C}(\mathfrak{g})$ is an ideal of $\mathrm{g}$.

(ii) $\operatorname{Ad}(\exp t u) \mathcal{C}(\mathrm{g})=\mathcal{C}(\mathrm{g})$ for any $u \in \mathfrak{g}, t \in \mathbf{R}$.

(iii) $\mathcal{C}(\mathfrak{g})$ has the property $(\mathrm{P})$ replacing $\mathfrak{h}$ by $\mathcal{G}(\mathrm{g})$.

(iv) $\int_{a}^{b} \operatorname{Ad}(\exp t u) v d t \in \mathcal{C}(\mathfrak{g})$ for any $u \in \mathfrak{g}, v \in \mathcal{C}(\mathrm{g})$.

Proof. (iii) is trivial by definition.

(i) Let $w=\sum_{\alpha}\left[u_{\alpha}, v_{\alpha}\right], u_{\alpha} \in \mathcal{C}(\mathfrak{g}), v_{\alpha} \in \mathfrak{g}$, be an element of $[\mathcal{C}(\mathfrak{g}), g]$. Since $\mathcal{C}(\mathfrak{g})$ has property $(\mathrm{P})$, there are compact sets $K_{u_{a}}$. Set $K_{w}=\cup K_{u_{a}}$. Then $K_{w} \cap \Sigma_{0}([\mathcal{C}(g), g])=\varnothing$ because of Lemma 1.4. So, it is easy to check that $[\mathcal{C}(g), g]$ has property $(P)$; hence by the maximality we have $[\mathcal{C}(g), g] \subset \mathcal{C}(g)$.

(ii) Let $\varphi=\exp t u$. It is easy to see that $\Sigma_{0}(\operatorname{Ad}(\varphi) \mathcal{C}(g))=\varphi \Sigma_{0}(\mathcal{C}(g))$. For any $v \in \mathcal{C}(\mathrm{g})$, there is a compact set $K_{v}$ stated in property (P). We set 
$K_{\mathrm{Ad}(\varphi) \nu}=\varphi K_{\nu}$. It is now easy to check that $\mathrm{Ad}(\varphi) \mathcal{C}(\mathrm{g})$ has property $(\mathrm{P})$, and hence $\operatorname{Ad}(\varphi) \mathcal{C}(\mathfrak{g}) \subset \mathcal{C}(\mathfrak{g})$, but this implies $\operatorname{Ad}(\varphi) \mathcal{C}(\mathfrak{g})=\mathcal{C}(\mathfrak{g})$ because the same is true for $\varphi^{-1}$.

We remark that the above equality also shows that $\varphi \Sigma_{0}(\mathcal{C}(g))=\Sigma_{0}(\mathcal{C}(g))$.

(iv) Let $\mathfrak{h}$ be the linear space of the finite sum of elements $\int_{c}^{d} \operatorname{Ad}(\exp t u) v d t$, $v \in \mathcal{C}(\mathrm{g})$, where $u \in g$ is arbitrarily fixed and $c, d$ are arbitrary. It is enough to show that $\mathcal{C}(\mathfrak{g})+\mathfrak{h}$ satisfies condition $(\mathrm{P})$. Remark that $\Sigma_{0}(\mathcal{C}(\mathfrak{g})+\mathfrak{h})$ $\subset \Sigma_{0}(\mathcal{C}(\mathfrak{g}))$. For any $v \in \mathcal{C}(\mathfrak{g})$, there is a compact set $K_{v}$ stated in property (P). Since $K_{\nu} \cap \Sigma_{0}(\mathcal{C}(\mathrm{g}))=\varnothing$ and $\Sigma_{0}(\mathcal{C}(\mathrm{g}))$ is invariant under the group \& generated by $\left\{\exp t u ; u \in g\right.$ \}, we see that $K_{w}=U_{t \in[c, d]} \varphi_{t} K_{\nu}$ is compact and $K_{w} \cap \Sigma_{0}(e(g))=\varnothing$, where $\varphi_{t}=\exp t u$. Since $[c, d] \times K_{v}$ is compact, for any $\varepsilon>0$ there is $\delta>0$ and an integer $m$ such that $\rho\left(\varphi_{t}(p), \varphi_{s}(q)\right)<\varepsilon$ whenever $p, q \in K_{v}, \rho(p, q)<\delta$ and $t, s \in[c, d],|t-s|<1 / m$, where $\rho$ means the distance. By property (P), $v$ can be written as a finite sum $v=\Sigma_{\alpha} v_{\alpha}$ such that $v_{\alpha} \in \mathcal{C}(\mathfrak{g}), \operatorname{supp} v_{\alpha} \subset K_{v}$ and diam $\left(\operatorname{supp} v_{\alpha}\right)<\delta$. Divide the interval $[c, d]$ into many small intervals $\left\{I_{j}\right\}$ so that the diameter of each $I_{j}$ is less than $1 / \mathrm{m}$. Now, we have

$$
\int_{c}^{d} \operatorname{Ad}(\exp t u) v d t=\sum_{\alpha} \sum_{j} \int_{l_{j}} \operatorname{Ad}(\exp t u) v_{\alpha} d t
$$

and

$$
\operatorname{supp} \int_{I_{j}} \operatorname{Ad}(\exp t u) v_{\alpha} d t \subset K_{w}
$$

Moreover,

$$
\operatorname{diam}\left(\operatorname{supp} \int_{I_{j}} \operatorname{Ad}(\exp t u) v_{\alpha} d t\right)<\varepsilon
$$

Using the above result, we get easily that $\mathcal{C}(\mathfrak{g})+\mathfrak{h}$ has property $(\mathrm{P})$.

ReMark. Property (ii) holds for any diffeomorphism $\varphi$ of $M$ such that $\operatorname{Ad}(\varphi) \mathfrak{g}=g$.

Corollary 1.1. If g satisfies $(\mathrm{C}, 5)$, then $\Sigma_{0}(\varrho(\mathrm{g}))=\varnothing$ or $\boldsymbol{M}$.

Proof. The group \& generated by $\{\exp t u ; u \in \mathfrak{g}\}$ acts transitively on $M$, but the set $\Sigma_{0}(e(g))$ is (S)-invariant.

Definition. An ideal $\mathfrak{h}$ of $\mathfrak{g}$ will be called a subcore of $g$ if $\mathfrak{h}$ has the following properties:

(i) $\operatorname{Ad}(\exp t u) \mathfrak{h}=\mathfrak{h}$ for any $u \in \mathfrak{g}, t \in \mathbf{R}$.

(ii) $\mathfrak{h}$ has property (P).

(iii) $\int_{a}^{b} \operatorname{Ad}(\exp t u) v d t \in \mathfrak{h}$ for any $u \in \mathfrak{g}, v \in \mathfrak{h}$.

If $g$ is a module over $C^{\infty}$ functions on $M$ with $\Sigma_{0}(\mathfrak{g})=\varnothing$, then the core $Q_{(g)}$ is the totality of $u \in \mathfrak{g}$ with compact support. Let $S$ be a $\mathbb{S}$-invariant 
subset of $M$ and let

$\mathfrak{h}=\{u \in g ;$ supp $u$ is compact, $u \equiv 0$ on a neighborhood of $S\}$.

Then $\mathfrak{h}$ is an ideal and a module over $C^{\infty}$ functions on $\dot{M}$. In fact, $\mathfrak{h}$ is a subcore of $\mathfrak{g}$.

2. Some remarks on the conditions $(C, 2)$ and $(C, 3)$, and $g$-orbits. Let $g$ be a Lie algebra of $C^{\infty}$ vector fields on $M$ with the properties $(C, 1)-(C, 3)$. Let $\Gamma\left(T^{p, q}\right)$ be the space of all $p$-covariant and $q$-contravariant tensor fields on $M$. The group of diffeomorphisms acts naturally on $\Gamma\left(T^{p, q}\right)$ and hence we have the notion of Lie derivatives $\mathfrak{I}_{u}: \Gamma\left(T^{p, q}\right) \rightarrow \Gamma\left(T^{p, q}\right)$ for any vector field $u$.

Proposition 2.1. The following Lie subalgebras of g satisfy $(\mathrm{C}, 1)-(\mathrm{C}, 3)$ :

(i) $\mathfrak{g}_{T}=\left\{u \in \mathfrak{g} ; \mathfrak{l}_{u} T=0\right\}$ for any $T \in \Gamma\left(T^{p, q}\right)$.

(ii) $\mathfrak{g}_{\mathbf{S}}=\left\{u \in g ; \mathfrak{R}_{u} \mathbf{S} \subset \mathbf{S}\right\}$, where $\mathbf{S}$ is a linear space of $\Gamma\left(T^{p, q}\right)$ with one of the following properties:

(a) $\mathbf{S}$ is spanned over the real numbers by a finite number of $T_{1}, \ldots, T_{N}$ $\in \Gamma\left(T^{p, q}\right)$ such that, for any $p \in M$, there is $k$ satisfying that $j_{p}^{k} T_{1}, \ldots, j_{p}^{k} T_{N}$ are linearly independent, where $j_{p}^{k}$ is the kth jet at $p$.

(b) $\mathbf{S}$ is a module over the $C^{\infty}$ functions on $M$ generated by $T_{1}, \ldots, T_{N}$ $\in \Gamma\left(T^{p, q}\right)$ such that $\mathbf{S}$ is closed in the $C^{\infty}$ topology.

Proof. Obviously, $g_{T}$ and $g_{S}$ satisfy $(C, 1)$. As for $(C, 2)$, we give the proof for the case (ii)(b). Others are much easier by the same proof. Let $T_{1}, \ldots, T_{N}$ be generators of $\mathbf{S}$. Obviously, $u \in g$ is contained in $g_{S}$ if and only if $\mathfrak{S}_{u} T_{i} \in \mathrm{S}$. Hence there is an $N$-by- $N$ matrix $A$ of $C^{\infty}$ functions such that $\mathfrak{L}_{u} T_{i}=\sum A_{i j} T_{j}$, where $A=\left(A_{i j}\right)$. Let $\varphi_{t}$ be the one parameter group generated by $u$ and $\varphi_{t}^{*}$ the natural action of $\varphi_{t}$ onto $\Gamma\left(T^{p, q}\right)$. By the definition of Lie derivatives, we have

$$
\frac{d}{d t}\left(\varphi_{t}^{*} T_{i}\right)(x)=\varphi_{t}^{*}\left(\mathfrak{L}_{u} T_{i}\right)(x)=\sum A_{i j}\left(\varphi_{t}(x)\right)\left(\varphi_{t}^{*} T_{j}\right)(x) .
$$

Consider the equation of the $N$-by- $N$ matrix $X(t, x)=\left(X_{i j}(t, x)\right)$,

$$
\frac{d}{d t} X(t, x)=A\left(\varphi_{t}(x)\right) X(t, x), \quad X(0, x) \equiv \mathrm{id},
$$

where $x \in M$ is regarded as a parameter. So, equation (*) has a unique solution $X(t, x)$ which is of $C^{\infty}$ with respect to $(t, x)$. Obviously, we have $\left(\varphi_{i}^{*} T_{i}\right)(x)=\sum_{j} X_{i j}(t, x) T_{j}(x)$; hence $\varphi_{t}^{*} T_{i} \in \mathbf{S}$. Thus, $\varphi_{i}^{*} \mathbf{S}=\mathbf{S}$ for any $u$ $\in \mathfrak{g}_{\mathbf{S}}$.

On the other hand, we have

$$
s^{-1}\left\{\varphi_{t}(\exp s v) \varphi_{t}^{-1}-1\right\}^{*} S \subset \mathbf{S} \text { for any } v \in g_{S}
$$


where $\varphi_{t}=\exp t u, u \in \mathrm{g}_{\mathrm{S}}$. By the closedness of $\mathrm{S}$, the limit $s \rightarrow 0$ is still contained in $\mathbf{S}$. Therefore, $\mathfrak{R}_{\operatorname{Ad}\left(\varphi_{1}\right)} \mathbf{S} \subset \mathbf{S}$. Since $\operatorname{Ad}\left(\varphi_{t}\right) \nu \in \mathfrak{g}$ by the assumption, we have $\operatorname{Ad}\left(\varphi_{t}\right) v \in \mathrm{g}_{\mathbf{S}}$. Thus, $\mathrm{g}_{\mathbf{S}}$ has property $(\mathrm{C}, 2)$.

For the proof that $g_{T}$ has property $(C, 3)$, we have only to note that $\mathfrak{R}_{u} T=0$ is a differential operator of order one with respect to $u$. Then it is easy to get the result. For cases (ii)(a) and (b), it is also easy to see that $g_{S}$ satisfies (LC) because of the closedness of $\mathbf{S}$.

Let $u$ be a $C^{\infty}$ vector field with a compact support such that, for any $p \in M$ and $k \geqslant 0$, there is $v_{p}^{k} \in g_{S}$ satisfying $j_{p}^{k} u=j_{p}^{k} v_{p}^{k}$. By the assumption, we have $u \in g$. Now, we consider case (ii)(a). Assume that $j_{p}^{k} T_{1}, \ldots, j_{p}^{k} T_{N}$ are linearly independent. Then there is an open neighborhood $V$ of $p$ on which $j^{k} T_{1}, \ldots, j^{k} T_{N}$ are linearly independent. Note that

$$
j_{q}^{r}\left(L_{u} T_{i}\right)=j_{q}^{r}\left(\mathfrak{Q}_{u_{q}^{++1}} T_{i}\right)=\sum_{j} \lambda_{j, q,}^{i} j_{q}^{r} T_{j},
$$

where $\lambda_{j, q, r}^{i}$ are constants. If $r \geqslant k$, then $\lambda_{j, q, r}^{i}$ are uniquely determined by $j_{q}^{k+1} u$. Hence, these are independent from $r$, and are $C^{\infty}$ functions of $q$ on $V$. Since $j^{r+1}\left(\mathfrak{R}_{u} T_{i}\right)=j^{1} j^{r}\left(\mathfrak{L}_{u} T_{i}\right)$, we have

$$
\sum_{j}\left(j_{q}^{l} \lambda_{j, q}^{i}\right)\left(j_{q}^{r} T_{j}\right) \equiv 0 \text { on } V,
$$

but this means that $\lambda_{j, q}^{i}$ are constant on $V$. Thus, we get $j^{k}\left(\mathfrak{R}_{u} T_{i}-\Sigma \lambda_{i}^{j} T_{j}\right)$ $\equiv 0$ on $V$; hence $\mathfrak{L}_{u} T_{i} \equiv \Sigma \lambda_{i}^{j} T_{j}$ on $V$. Since $M$ is connected, we have that $u \in \mathrm{g}_{\mathbf{s}}$.

Now, consider case (ii)(b). Since $j_{p}^{r}\left(\mathfrak{R}_{u} T_{i}\right)=j_{p}^{r}\left(\mathfrak{R}_{v^{r+1}} T_{i}\right) \in j_{p}^{r} S$ for any $r \geqslant 0$, we have $\mathfrak{R}_{u} T_{i} \in \overline{\mathbf{S}}$ by Corollary 1.7 of $[5, \mathrm{p} .25]$. Since $\overline{\mathbf{S}}=\mathbf{S}$, we have the desired result.

Let $g_{\gamma}, \gamma \in \Lambda$, be Lie algebras with $(C, 1)$ and $(C, 2)$. Then, it is easy to see that $g=\bigcap_{\gamma \in \Lambda} g_{\gamma}$ satisfies $(C, 1)$ and $(C, 2)$. The following is also a simple remark, but this will be used later:

LeMma 2.1. Let $\mathfrak{g}$ be a Lie algebra with $(\mathrm{C}, 1)$ and $(\mathrm{C}, 2)$ and $\mathfrak{h}$ a closed finite codimensional subalgebra of $\mathfrak{g}$. Then $\mathfrak{h}$ satisfies $(\mathrm{C}, 1)$ and $(\mathrm{C}, 2)$. Moreover, if $\mathfrak{h}$ is an ideal, then $\operatorname{Ad}(\exp t u) \mathfrak{h}=\mathfrak{h}$ for any $u \in \mathfrak{g}$.

Proof. $\mathfrak{g} / \mathfrak{h}$ is a finite dimensional space and the projection $\pi: \mathfrak{g} \rightarrow \mathfrak{g} / \mathfrak{h}$ is continuous. For any $u \in \mathfrak{h}$, ad $(u): \mathfrak{g} \rightarrow \mathfrak{g}$ leaves $\mathfrak{h}$ invariant. Therefore $u$ induces a linear map $A(u): \mathfrak{g} / \mathfrak{h} \rightarrow \mathfrak{g} / \mathfrak{h}$ such that $A(u) \pi=\pi$ ad $(u)$. We consider $\pi(\operatorname{Ad}(\exp t u) v)$ and $e^{t A(u)} \pi v$ for any $v \in \mathfrak{g}$. The first one satisfies

$$
\frac{d}{d t} \pi(\operatorname{Ad}(\exp t u) v)=\pi[u, \operatorname{Ad}(\exp t u) v]=A(u) \pi(\operatorname{Ad}(\exp t u) v) .
$$

The second satisfies 


$$
\frac{d}{d t} e^{t A(u)} \pi v=A(u) e^{t A(u)} \pi v .
$$

These are the same equations. Therefore, we have $\pi(\operatorname{Ad}(\exp t u) v)=e^{t A(u)} \pi v$, because the initial conditions are the same. If $v \in \mathfrak{h}$, then $\pi v=0$. Therefore, Ad $(\exp t u) v \in \mathfrak{h}$.

If $\mathfrak{h}$ is an ideal of $\mathfrak{g}$, then we can use any $u \in \mathfrak{g}$ instead of $u \in \mathfrak{h}$ in the above argument; hence we have the desired result.

Let $g$ be a Lie algebra with $(C, 1)$ and $(C, 2)$, and 8 the group generated by $\{\exp t u ; u \in g\}$. We will show next that the orbit $\&(p)$ of $p \in M$ is an immersed submanifold of $M$. Let $m=\operatorname{dim}\{u(p) ; u \in g\}$. We choose $m$ vector fields $v_{1}, \ldots, v_{m} \in g$ such that $v_{1}(p), \ldots, v_{m}(p)$ are linearly independent. Define a $C^{\infty}$ mapping $\mathcal{E}_{p}$ of $\mathbf{R}^{m}$ into $M$ by

$$
\mathcal{E}_{p}\left(t_{1}, \ldots, t_{m}\right)=\exp \left(t_{1} v_{1}+\cdots+t_{m} v_{m}\right)(p) .
$$

Obviously, the derivative $\left(d \varepsilon_{p}\right)_{0}$ at the origin is of maximal rank; hence there is a neighborhood $U$ of 0 of $\mathbf{R}^{m}$ such that $\mathscr{E}_{p}(U)$ is an $m$-dimensional submanifold of $M$.

LEMMA 2.2. The tangent space $T_{q} \mathcal{E}_{p}(U)$ of $q \in \mathcal{E}_{p}(U)$ is given by $g(q)$ $=\{u(q) ; u \in g\}$.

Proof. Let $q=\mathcal{E}_{p}\left(\tau_{1}, \ldots, \tau_{m}\right)$, i.e., $q=\varphi(p), \varphi=\exp w$ and $w=\tau_{1} \nu_{1}$ $+\cdots+\tau_{m} v_{m} \cdot\left(d \mathscr{E}_{p}\right)_{\left(\eta_{1}, \ldots, \tau_{m}\right)} \mathbf{R}^{m}$ is the tangent space of $\mathscr{E}_{p}(U)$ at $q$. Since $d \varphi g(p)=g(q)$, we have only to show that $d \varphi^{-1}\left(d \varepsilon_{p}\right)_{\left(\eta_{1}, \ldots, \tau_{m}\right.} \mathbf{R}^{m}=g(p)$.

Set $\varphi_{t}=\exp t w$ and consider $d \varphi_{t}^{-1}\left(d E_{p}\right)_{t\left(\eta_{1}, \ldots, \tau_{m}\right)} t X$, where $X=\left(\theta_{1}, \ldots\right.$, $\left.\theta_{m}\right) \in \mathbf{R}^{m}$. Set $v=\theta_{1} v_{1}+\cdots+\theta_{m} v_{m}$. Then

$$
d \varphi_{t}^{-1}\left(d E_{p}\right)_{t\left(\tau_{1}, \ldots, \tau_{m}\right)} t X=\left.d \varphi_{t}^{-1} \frac{\partial}{\partial s}\right|_{s=0} \exp t(w+s v)(p) .
$$

If $t=0$, then this is zero. Hence

$$
\begin{gathered}
\left.d \varphi^{-1} \frac{\partial}{\partial s}\right|_{s=0} \exp (w+s v)(p)=\left.\frac{\partial}{\partial s}\right|_{s=0} \int_{0}^{1} \frac{\partial}{\partial t} \varphi_{t}^{-1} \exp t(w+s v)(p) d t \\
=\left.\int_{0}^{1} \frac{\partial}{\partial s}\right|_{s=0}\left\{-d \varphi_{t}^{-1} w(\exp t(w+s v)(p))\right. \\
\left.\quad+d \varphi_{t}^{-1}(w+s v)(\exp t(w+s v)(p))\right\} d t \\
=\left.\int_{0}^{1} \frac{\partial}{\partial s}\right|_{s=0} s d \varphi_{t}^{-1} v(\exp t(w+s v)(p)) d t \\
=\int_{0}^{1} d \varphi_{t}^{-1} v\left(\varphi_{t}(p)\right) d t=\int_{0}^{1}\left(\operatorname{Ad}\left(\varphi_{t}^{-1}\right) v\right)(p) d t .
\end{gathered}
$$


Since $\left(\operatorname{Ad}\left(\varphi_{t}^{-1}\right) v\right)(p) \in g(p)$, we have $\int_{0}^{1}\left(\operatorname{Ad}\left(\varphi_{t}^{-1}\right) v\right)(p) d t \in g(p)$. Since both sides have the same dimension, we have

$$
d \varphi^{-1}\left(d \varepsilon_{p}\right)_{\left(\tau_{1}, \ldots, \tau_{m}\right)} \mathbf{R}^{m}=g(p) .
$$

The above lemma shows that any $u \in g$ defines a tangent vector field on $\mathscr{E}_{p}(U)$. Therefore, if we make another $\mathcal{E}_{q}\left(U^{\prime}\right)$ for $q \in \mathcal{E}_{p}(U)$ by the same procedure, then $\varepsilon_{p}(U) \cup \delta_{q}\left(U^{\prime}\right)$ is a connected submanifold of $M$. We make such $\varepsilon_{q}\left(U^{\prime}\right)$ at every $q$ of the $\circlearrowleft$-orbit $\&(p)$. Then, we see

$$
\mathbb{S}(p)=\bigcup\left\{\mathcal{E}_{q}\left(U^{\prime}\right) ; q \in \mathbb{S}(p)\right\}
$$

and hence $\mathbb{S}(p)$ is an immersed submanifold of $M$ such that the tangent space $T_{q} \mathbb{S}(p)$ is given by $\mathbb{S}(q)(\mathrm{cf}$. [13, Theorem 4.1]). This orbit $\mathbb{S}(p)$ will be called a g-orbit of $p$.

Now, let $\mathrm{g}$ be a Lie algebra with conditions $(\mathrm{C}, 1)$ and $(\mathrm{C}, 2)$, and $N$ be a $\mathrm{g}$ orbit. We set $m=\operatorname{dim} N, n=\operatorname{dim} M$. For any $p \in N$, there is a $C^{\infty}$ diffeomorphism $\eta$ of a neighborhood $V$ of the origin 0 of $\mathbf{R}^{n-m}$ into $M$ such that $\eta(0)=p$ and $\eta(V)$ is a submanifold of $M$ which is transversal to $N$ at $p$. Let $v_{1}, \ldots, v_{m}$ be elements of $g$ such that $v_{1}(p), \ldots, v_{m}(p)$ are linearly independent. We make a $C^{\infty}$ mapping $\mathscr{E}_{V}$ of $V \times \mathbf{R}^{m}$ into $M$ by

$$
\varepsilon_{V}\left(x, t_{1}, \ldots, t_{m}\right)=\exp \left(t_{1} v_{1}+\cdots+t_{m} v_{m}\right)(\eta(x)) .
$$

This is of maximal rank at $(0,0)$; hence there are open, contractible neighborhoods $W, U$ of the origins of $\mathbf{R}^{n-m}, \mathbf{R}^{m}$ respectively such that $W \subset V$ and the restriction of $\varepsilon_{V}$ onto $W \times U$ is a $C^{\infty}$ diffeomorphism onto a neighborhood of $p \in M$. Note that if $q=\eta(x), x \in W$, is a point of $N$, then $\varepsilon_{V}(x, U)$ is a neighborhood of $\eta(x)$ in the manifold $N$. Thus, we have the following existence of slice box.

Lemma 2.3. Let $N$ be a g-orbit. For any point $p \in N$, there is a $C^{\infty}$ diffeomorphism $\varepsilon_{V}: W \times U \rightarrow M$ such that $\varepsilon_{V}(0,0)=p$ and for each $q \in N$ $\cap \varepsilon_{V}(W \times U)$ there exists $x \in W$ such that $\mathcal{E}_{V}(x, U)$ is a neighborhood of $q$ in $N$.

Proof. For any $q \in N \cap \varepsilon_{V}(W \times U)$, there exists $x \in W$ such that $\varepsilon_{V}(x, U) \ni q$. Thus, we have only to show that $\varepsilon_{V}(x, U) \subset N$. By Lemma 2.2, we know that any integral curve $(\exp t u)(q)$ of $u \in \mathfrak{g}$ with the initial point $q$ is contained in $\mathcal{E}_{V}(x, U)$ for sufficiently small $t$. Therefore, $\mathcal{E}_{V}(x, U)$ is contained in the g-orbit $N$. Since $\varepsilon_{V}(x, *): U \rightarrow N$ is of maximal rank, we see that $\delta_{V}(x, U)$ is an open subset of $N$. This proves the lemma.

Let $g$ be a Lie algebra on $M$ with properties $(C, 1),(C, 2)$ and the following:

$$
\int_{a}^{b} \operatorname{Ad}(\exp t u) v d t \in \mathfrak{g} \quad \text { for any } u, v \in \mathfrak{g} .
$$


If any $u \in g$ has a compact support, then conditions $(C, 1)-(C, 3)$ yield property $(\mathrm{IC}, 2)^{\prime}$, because of Lemma 1.2. So, cores of Lie algebras with $(\mathrm{C}, 1)-(\mathrm{C}, 4)$ have properties $(\mathrm{C}, 1),(\mathrm{C}, 2)$ and $(\mathrm{IC}, 2)$.

Let $N$ be a $g$-orbit of $p \in M$, and $\mathrm{g}_{N}$ the restriction of $g$ onto the immersed submanifold $N . N$ is, of course, invariant under the group (5) generated by $\{\exp t u ; u \in \mathfrak{g}\}$.

LEMMA 2.4. Notations and assumptions being as above, the restriction $g_{N}$ has properties $(\mathrm{C}, 1),(\mathrm{C}, 2)$ and $(\mathrm{IC}, 2)^{\prime}$.

Proof is easy by definitions.

REMARK. The restriction of a riemannian metric on $M$ onto $N$ is not complete in general. So, the restriction $\mathcal{C}(g)_{N}$ of the cure of $g$ may not be the core of $g_{N}$.

3. Maximality of isotropy subalgebras of a core. Let $g$ be a Lie algebra of $C^{\infty}$ vector fields on $M$ with properties $(\mathrm{C}, 1),(\mathrm{C}, 2),(\mathrm{IC}, 2)$ and $(\mathrm{C}, 4)$ in the introduction, and $\mathbb{E}$ the core of $g$. For a point $p \in M-\Sigma_{0}(\mathbb{E})$, we denote by $\mathfrak{F}_{p}$ the isotropy subalgebra of $\mathbb{E}$ at $p$. The purpose of this section is to show that $\mathbb{E}_{p}$ is a maximal subalgebra of $\mathbb{E}$.

Now, $\mathbb{E}$ has the properties $(\mathrm{C}, 1),(\mathrm{C}, 2)$ and $(\mathrm{IC}, 2)^{\prime}$ of the previous section. Let $N$ be a $\mathfrak{}$-orbit of $p \in M-\Sigma_{0}(\mathfrak{E})$. We fix an arbitrary complete $C^{\infty}$ riemannian metric on $N$. The following are the properties of the restriction $\mathbb{E}_{N}$ of $\mathbb{E}$ which will be used in this paper:

Proposition 3.1. (i) Every $u \in \mathfrak{E}_{N}$ is complete.

(ii) $\operatorname{Ad}(\exp t u) \mathfrak{S}_{N}=\mathfrak{E}_{N}$ for any $u \in \mathfrak{E}_{N}, t \in \mathbf{R}$.

(iii) $\int_{a}^{b} \operatorname{Ad}(\exp t u) v d t \in \mathfrak{F}_{N}$ for any $u, v \in \mathfrak{F}_{N},-\infty<a \leqslant b<\infty$.

(iv) Let $K, q$ be a compact set of $N$ and a point of $N$, respectively, such that $K \boxplus q$. Let $\varepsilon$ be an arbitrary positive number. For any $u \in \mathbb{E}_{N}$ such that $u(q) \neq 0$, there is $v \in \aleph_{N}$ with the following properties:

(a) $v \equiv u$ on a neighborhood of $q$.

(b) $\nu \equiv 0$ on a neighborhood of $K$.

(c) The diameter of the connected component of supp $v$ containing $q$ is less than $\varepsilon$, where the diameter is given by using an arbitrarily fixed complete riemannian metric on $N$.

Proof. (i), (ii) and (iii) have been proved in Lemma 2.4. As for (iv), we use Lemma 2.3 and property (P) of cores. Then it is not hard to get the desired result.

Let $\aleph_{p}$ be the isotropy subalgebra of $\Subset$ at $p \in M-\Sigma_{0}(\Subset)$, and $\mho_{N, p}$ the restriction of $\mathfrak{\mho}_{p}$ onto $N$. $\mathfrak{\mho}_{N, p}$ is the isotropy subalgebra of $\mathfrak{F}_{N}$ at $p \in N$. Let $\mathfrak{h}^{\prime}$ be a subalgebra of $\mathfrak{夭}_{N}$ such that $\mathfrak{\Im}_{N, p} \varsubsetneqq \mathfrak{h}^{\prime} \varsubsetneqq \mathfrak{\Im}_{N}$. We set $\mathfrak{h}=\left\{u \in \mathfrak{c} ;\left.u\right|_{N}\right.$ $\left.\in \mathfrak{h}^{\prime}\right\}$. Then $\mathfrak{h}$ is a subalgebra of $\mathfrak{E}$ such that $\mathfrak{E}_{p} \subsetneq \mathfrak{h} \subsetneq \mathfrak{c}$. The converse is also true. 
Now, since $\mathfrak{h} \supset \mathfrak{\mho}_{p}$ and $\mathfrak{C}_{p}$ is a closed finite codimensional subspace of $\mathfrak{\mho}$ in the $C^{\infty}$ topology, we see that $\mathfrak{h}$ is a closed finite codimensional subalgebra of $\mathfrak{夭}$. By the same reasoning, we see also that any subalgebra $\mathfrak{h}^{\prime}$ of $\mathfrak{E}_{N}$ such that $\mathfrak{h}^{\prime} \supset \mathfrak{\complement}_{N, p}$ is a closed finite codimensional subalgebra. Hence by Lemma 2.1, we have that $\operatorname{Ad}(\exp t u) \mathfrak{h}^{\prime}=\mathfrak{h}^{\prime}$ for any $u \in \mathfrak{h}^{\prime}$. By Lemma 2.2 and remarks there, we can define $\mathfrak{h}^{\prime}$-orbits in the manifold $N$.

THEOREM 3.1. Let $N$ be a $\mathbb{E}$-orbit of $p \in M-\Sigma_{0}(\mathbb{\mho})$. Then the isotropy

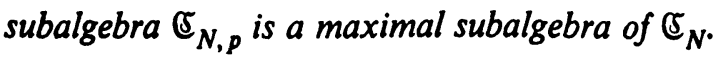

Proof. Let $\mathfrak{h}$ be a subalgebra of $\mathfrak{夭}_{N}$ such that $\mathfrak{夭}_{N, p} \subsetneq \mathfrak{h} \subseteq \mathfrak{夭}_{N}$. Let $\mathfrak{X}$ be the group generated by $\{\exp t u ; u \in \mathfrak{h}\}$. The h-orbit of $p$ is in fact given by $\mathscr{S C}(p)$. For any $q \in \mathcal{K}(p)$ such that $q \neq p$, there is $u \in \mathbb{E}_{N}$ such that $u(q) \notin \mathfrak{h}(q)$. Remark that $\mathfrak{h}(q)$ is the tangent space of $\mathfrak{I C}(p)$ at $q$ (cf. Lemma 2.2). By Proposition 3.1, there is $v \in \mathbb{E}_{N}$ such that $v(q)=u(q)$ and $v=0$ on a neighborhood of $p$. We set $\varphi_{t}=\exp t v$. Then, $\varphi_{t} \mathcal{S C}(p) \neq \mathcal{S C}(p)$ for some $t$, because otherwise we have $\operatorname{dim} \mathcal{H C}(p)>\operatorname{dim} \mathfrak{h}(q)$. Remark, however, that $v \in \mathfrak{E}_{N, p} \subset \mathfrak{h}$; hence $\operatorname{Ad}\left(\varphi_{t}\right) \mathfrak{h}=\mathfrak{h}$. So, we have $\varphi_{t} \mathcal{H C}_{\varphi_{t}}^{-1}=\mathscr{C}$, because $\exp \operatorname{Ad}\left(\varphi_{t}\right) w=\varphi_{t}(\exp w) \varphi_{t}^{-1}$. Since $\varphi_{t}^{-1}(p)=p$, the above equality shows that $\varphi_{t} \mathcal{H}(p)=\mathscr{H C}(p)$. This is a contradiction and hence we have $\mathbb{E}_{N, p}$ is a maximal subalgebra of $\mathbb{E}_{N}$.

4. Proper maximal ideals of a subcore. Let $g$ be a Lie algebra of $C^{\infty}$ vector fields on $M$ with conditions (C,1), $(\mathrm{C}, 2),(\mathrm{IC}, 2)$ and $(\mathrm{C}, 4)$ in the introduction. Let $\mathfrak{h}$ be a nontrivial subcore of $g$. Throughout this section, $g$ means always a proper maximal ideal of $\mathfrak{h}$ such that $g D[\mathfrak{h}, \mathfrak{h}]$. We denote by $B(p, \varepsilon)$ the $\varepsilon$ neighborhood of $p$ in $M$ by an arbitrarily fixed complete riemannian metric on $M$. By $\mathfrak{h}_{B(p, \varepsilon)}^{0}$, we mean the totality of $u$ such that $u \equiv 0$ on $B(p, \varepsilon)$.

\section{Lemma 4.1. There is a point $p \in M$ such that $g \supset \mathfrak{h}_{B(p, \varepsilon)}^{0}$.}

Proof. Suppose $\mathfrak{h}=g+\mathfrak{h}_{B(p, \varepsilon)}^{0}$ for any point $p \in M$. By property (P), any $u, v \in \mathfrak{h}$ can be written as finite sums $u=\Sigma_{\alpha} u_{\alpha}, v=\Sigma_{\beta} v_{\beta}$ such that $u_{\alpha}$, $v_{\beta} \in \mathfrak{h}$ and $\operatorname{diam}\left(\operatorname{supp} u_{\alpha}\right)<\varepsilon / 2, \operatorname{diam}\left(\operatorname{supp} v_{\beta}\right)<\varepsilon / 2$. Thus, $[u, v]$ $=\sum\left[u_{\alpha}, v_{\beta}\right]$ where the summation runs through all pairs $(\alpha, \beta)$ such that $\operatorname{supp} u_{\alpha} \cap \operatorname{supp} v_{\beta} \neq \varnothing$. Note that if $\operatorname{supp} u_{\alpha} \cap \operatorname{supp} v_{\beta} \neq \varnothing$, then there is a point $p \in M$ such that $B(p, \varepsilon)$ contains $\operatorname{supp} u_{\alpha} \cup \operatorname{supp} v_{\beta}$. Since $\mathfrak{h}=9$ $+\mathfrak{h}_{B(p, \varepsilon)}^{0}$, we see that there is $\bar{u}_{\alpha} \in \mathcal{G}, w_{\alpha} \in \mathfrak{h}_{B(p, \varepsilon)}^{0}$ such that $u_{\alpha}=\bar{u}_{\alpha}+w_{\alpha}$. Therefore $\left[u_{\alpha}, v_{\beta}\right]=\left[\bar{u}_{\alpha}, v_{\beta}\right] \in \mathscr{g}$. This means that $\mathcal{G} \supset[\mathfrak{h}, \mathfrak{h}]$, contradicting the assumption for $\mathscr{g}$. So, there is $p \in M$ such that $\mathfrak{h} \supsetneq \mathfrak{g}+\mathfrak{h}_{B(p, \varepsilon)}^{0}$. The right-hand side is an ideal of $\mathfrak{h}$. Therefore the maximality of $g$ shows the lemma.

We set $A_{\varepsilon}=\left\{p \in M ; \mathfrak{h}_{B(p, \varepsilon)}^{0} \subset g\right\}$.

LemMa 4.2. If $\varepsilon>\delta$, then $A_{\varepsilon} \supset \overline{A_{\delta}}$ (the closure of $A_{\delta}$ ). 
Proof. Let $p$ be a point in $\overline{A_{\delta}}$. There is $q \in A_{\delta}$ such that $\rho(p, q)<\varepsilon-\delta$. Then, obviously, $\mathfrak{h}_{B(p, \varepsilon)}^{0} \subset \mathfrak{h}_{B(q, \delta)}^{0} \subset 9$.

LEMMA 4.3. Notations and assumptions being as above, there is a unique point $p \in M-\Sigma_{0}(\mathfrak{h})$ such that $\mathfrak{g} \supset \mathfrak{h}_{p}^{V}$, where $\mathfrak{h}_{p}^{V}=\{u \in \mathfrak{h} ; u \equiv 0$ on a neighborhood of $p$ \}.

Proof. It is easy to see that, for a sufficiently small $\varepsilon, A_{\varepsilon}$ is contained in a compact subset of $M$. Thus, by the above lemma, there is a point $p$ $\in \cap_{\varepsilon}>0 A_{\varepsilon}$. Obviously $g \supset \mathfrak{h}_{B(p, \varepsilon)}^{0}$ for any $\varepsilon$. Hence we have $g \supset \mathfrak{h}_{p}^{V}$. Note that if $p \neq q$ then $\mathfrak{h}_{p}^{V}+\mathfrak{h}_{q}^{V}=\mathfrak{h}$ by property (P). Therefore such a point $p$ must be unique. Recall that any $u \in \mathfrak{h}$ is identically zero on a neighborhood of $\Sigma_{0}(\mathfrak{h})$. Therefore $\mathfrak{h}=\mathfrak{h}_{p}^{V}$ for any $p \in \Sigma_{0}(\mathfrak{h})$. Therefore the point $p$ obtained above cannot be in $\Sigma_{0}(\mathfrak{h})$.

Lemma 4.4. Every $u \in q$ vanishes at $p$.

Proof. If $u \in g$ does not vanish at $p$, then use Lemma 1.3, and we have $\mathfrak{g}=\mathfrak{h}$.

Let

$$
\mathfrak{h}_{p}^{\infty}=\left\{u \in \mathfrak{h} ;\left(\operatorname{ad}\left(w_{1}\right) \cdots \operatorname{ad}\left(w_{N}\right) u\right)(p)=0\right.
$$

for any $N$ and $\left.w_{1}, \ldots, w_{N} \in \mathfrak{h}\right\}$.

Lemma 4.5. Suppose $\mathscr{G} \supset \mathfrak{h}_{p}^{V}, p \in M-\Sigma_{0}(\mathfrak{h})$. Then $\mathfrak{g}=\mathfrak{h}_{p}^{\infty}$.

Proof. By the above lemma, we have $g \subset \mathfrak{h}_{p}^{\infty}$. Since $\mathfrak{h}_{p}^{\infty}$ is an ideal of $\mathfrak{h}$, we get $\mathrm{g}=\mathfrak{h}_{p}^{\infty}$ by the maximality.

Lemma 4.6. For any $p \in M-\Sigma_{0}(\mathfrak{h}), \mathfrak{h}_{p}^{\infty}$ is a maximal ideal of $\mathfrak{h}$ such that $\mathfrak{h}_{p}^{\infty} D[\mathfrak{h}, \mathfrak{h}]$.

Proof. By the above lemma, we see that $\mathfrak{h}_{p}^{\infty}$ is a maximal ideal of $\mathfrak{h}$. To show that $\mathfrak{h}_{p}^{\infty} D[\mathfrak{h}, \mathfrak{h}]$, we have only to remark that there is an element $w \in[\mathfrak{h}, \mathfrak{h}]$ such that $w(p) \neq 0$ (cf. Lemma 1.3).

Hence, we have

Proposition 4.1. There is a one-to-one correspondence of the points of $M-\Sigma_{0}(\mathfrak{h})$ onto the set of all maximal ideals 9 of $\mathfrak{h}$ such that $\mathfrak{g} D[\mathfrak{h}, \mathfrak{h}]$.

5. Continuity of the isomorphism $\Phi$ and the proof of Theorem $A$. We use the same notations as in the previous section. First of all, we will give the following characterization of zeros of $u$, which is essentially due to Pursell and Shanks [10]:

LeMMA 5.1. For any point $p \in M-\Sigma_{0}(\mathfrak{h}), u \in \mathfrak{h}$ does not vanish at $p$ if and only if $[u, \mathfrak{h}]+\mathfrak{h}_{p}^{\infty}=\mathfrak{h}$. 
Proof. If $u(p) \neq 0$, then Lemma 1.3 combined with property (P) shows that $[u, I(v)]-v \in \mathfrak{h}_{p}^{V} \subset \mathfrak{h}_{p}^{\infty}$.

Assume conversely that $[u, \mathfrak{h}]+\mathfrak{h}_{p}^{\infty}=\mathfrak{h}$. Assume furthermore that $u(p)$ $=0$. Then, we see that ad $(u) \mathfrak{h}_{p}^{k} \subset \mathfrak{h}_{p}^{k}$, where

$$
\begin{aligned}
\mathfrak{h}_{p}^{k}=\left\{u \in \mathfrak{h} ;\left(\operatorname{ad}\left(w_{1}\right) \cdots \operatorname{ad}\left(w_{j}\right) u\right)(p)\right. & =0 \\
\text { for any } 0 & \left.\leqslant j \leqslant k \text { and } w_{1}, \ldots, w_{j} \in \mathfrak{h}\right\} .
\end{aligned}
$$

We may assume that there is $k<\infty$ such that $\mathfrak{h}_{p}^{0} \neq \mathfrak{h}_{p}^{k}$ because otherwise, $u \in \mathfrak{h}_{p}^{\infty}$ and, hence, $[u, \mathfrak{h}]+\mathfrak{h}_{p}^{\infty}=\mathfrak{h}_{p}^{\infty} \subsetneq \mathfrak{h}$. We fix such $k$. The equality $[u, \mathfrak{h}]+\mathfrak{h}_{p}^{\infty}=\mathfrak{h}$ means that $\operatorname{ad}(u)$ induces a linear mapping $A(u)$ of $\mathfrak{h} / \mathfrak{h}_{p}^{k}$ onto itself; hence $A(u)$ must be a linear isomorphism, because $\operatorname{dim} \mathfrak{h} / \mathfrak{h}_{p}^{k}<\infty$.

Let $\tilde{u}$ be the equivalence class of $u$ in $\mathfrak{h} / \mathfrak{h}_{p}^{k}$. Then, $A(u) \tilde{u}=0$. Thus, we get $u \in \mathfrak{h}_{p}^{k}$. Therefore $\left[u, \mathfrak{h}_{p}^{0}\right] \subset \mathfrak{h}_{p}^{k}$. This means that $A(u) \mathfrak{h}_{p}^{0} / \mathfrak{h}_{p}^{k}=0$. This is a contradiction and hence we have $u(p) \neq 0$.

Let $\mathrm{g}$, ' $\mathrm{g}$ be Lie algebras of $C^{\infty}$ vector fields on $M$ and $N$, respectively, with conditions (C,1), (C,2), (IC,2) and (C,4). Let $\Phi$ be an isomorphism of $g$ onto ' $g$ such that $\Phi$ sends a nontrivial subcore $\mathfrak{h}$ to a subcore ' $\mathfrak{h}$.

For any point $p \in M-\Sigma_{0}(\mathfrak{h}), \mathfrak{h}_{p}^{\infty}$ is a maximal ideal of $\mathfrak{h}$ such that $\mathfrak{h}_{p}^{\infty} D[\mathfrak{h}, \mathfrak{h}]$. Hence, $\Phi\left(\mathfrak{h}_{p}^{\infty}\right)$ is a maximal ideal of 'hy with $\Phi\left(\mathfrak{h}_{p}^{\infty}\right) D\left[{ }^{\prime} \mathfrak{h}, \mathfrak{h}\right]$. By Proposition 4.1, there is a point $\varphi(p) \in N-\Sigma_{0}(\mathfrak{h})$ such that $\Phi\left(\mathfrak{h}_{p}^{\infty}\right)$ $=h^{\prime}(p) \cdot \varphi$ is obviously a one-to-one mapping of $M-\Sigma_{0}(\mathfrak{h})$ onto $N-\Sigma_{0}(\mathfrak{h})$.

Lemma 5.2. $\varphi: M-\Sigma_{0}(\mathfrak{h}) \rightarrow N-\Sigma_{0}(\mathfrak{h})$ is a homeomorphism.

The proof is seen in Pursell and Shanks [10, p. 471] or Koriyama [4, Proposition 4.7].

Now, $\Phi$ induces an isomorphism of $\mathfrak{h}$ onto ' $\mathfrak{h}$. By Lemma 5.1, we see that $\Phi\left(\mathfrak{h}_{p}^{0}\right)=\mathfrak{h}_{\varphi(p)}^{0}$ for any $p \in M-\Sigma_{0}(\mathfrak{h})$.

LEMMA 5.3. $\Phi: \mathfrak{h} \rightarrow \mathfrak{h}$ is continuous in the pointwise convergence topology.

Proof. $\Phi: \mathfrak{h} \rightarrow \mathfrak{h}$ induces a linear isomorphism $\tilde{\Phi}_{p}$ of $\mathfrak{h} / \mathfrak{h}_{p}^{0}$ onto $\mathfrak{h} / \mathfrak{h}_{\varphi(p)}^{0}$. This is continuous, because $\operatorname{dim} \mathfrak{h} / \mathfrak{h}_{p}^{0}<\infty$. For any $u \in \mathfrak{h}, \mathfrak{u}(p)$ is canonically identified with an element of $\mathfrak{h} / \mathfrak{h}_{p}^{0}$ and $\tilde{\Phi}_{p}(u(p))=\Phi(u)(\varphi(p))$. Therefore, if $\left\{w_{n}\right\}$ converges to $w \in \mathfrak{h}$ in the pointwise convergence topology, we have

$$
\lim _{n \rightarrow \infty} \Phi\left(w_{n}\right)(\varphi(p))=\lim _{n \rightarrow \infty} \tilde{\Phi}_{p}\left(w_{n}(p)\right)=\tilde{\Phi}_{p}(w(p))=\Phi(w)(\varphi(p))
$$

for any $p \in M-\Sigma_{0}(\mathfrak{h})$. Thus, $\lim _{n \rightarrow \infty} \Phi\left(w_{n}\right)(q)=\Phi(w)(q)$ for every $q \in N$ $-\Sigma_{0}(' \mathfrak{h})$. For a point $q \in \Sigma_{0}(' \mathfrak{h}), \Phi\left(w_{n}\right)(q)=\Phi(w)(q)=0$. Thus, we get the continuity.

Recall that a subcore $\mathfrak{h}$ has the property $\operatorname{Ad}(\exp t u) \mathfrak{h}=\mathfrak{h}$ for any $u \in \mathfrak{g}$.

Lemma 5.4. $\Phi(\operatorname{Ad}(\exp t u) v)=\operatorname{Ad}(\exp t \Phi(u)) \Phi(v)$ for any $u \in \mathfrak{g}$ and $v \in \mathfrak{h}$. 
Proof. The right-hand side satisfies the equation

$$
\frac{d}{d t} \operatorname{Ad}(\exp t \Phi(u)) \Phi(v)=[\Phi(u), \operatorname{Ad}(\exp t \Phi(u)) \Phi(v)] .
$$

Let $W(t, q)=\operatorname{Ad}(\exp t \Phi(u)) \Phi(v)(q), q \in N$. By using a local coordinate system $x_{1}, \ldots, x_{n}$ at $q \in N$, the above equation is written as

$$
\left(\frac{\partial}{\partial t}+\sum X_{j} \frac{\partial}{\partial x_{j}}\right) w_{i}(t, q)=\sum_{j} \frac{\partial X_{i}}{\partial x_{j}} w_{j}(t, q)
$$

where $\Phi(u)=\sum X_{i} \partial / \partial x_{i}, W=\sum_{j} w_{j} \partial / \partial x_{j}$.

Since

$$
(1 / \delta)\{\operatorname{Ad}(\exp (t+\delta) u) v-\operatorname{Ad}(\exp t u) v\}
$$

converges to $[u, \operatorname{Ad}(\exp t u) v]$ with $\delta \rightarrow 0$ in the pointwise convergence topology, we see that, for every point $q \in N$,

$$
\begin{aligned}
& \lim _{\delta \rightarrow 0} \frac{1}{\delta}\{\Phi(\operatorname{Ad}(\exp (t+\delta) u) v)-\Phi(\operatorname{Ad}(\exp t u) v)\}(q) \\
& =\Phi([u, \operatorname{Ad}(\exp t u) v])(q)=[\Phi(u), \Phi(\operatorname{Ad}(\exp t u) v)](q) .
\end{aligned}
$$

Put $\mathscr{W}(t, q)=\Phi(\operatorname{Ad}(\exp t u) v)(q)$. Then the above equality means that $\mathscr{W}(t, q)$ is differentiable with respect to $t$, and satisfies the same differential equation $(\alpha)$. Since $W(0, *)=\tilde{W}(0, *)$, we have the desired formula by the uniqueness of the solution of $(\alpha)$.

For any $u \in \mathfrak{g}$, we set $\psi_{t}=\exp t u, \tilde{\psi}_{t}=\exp t \Phi(u)$.

LEMMA 5.5. We have $\varphi \psi_{t}=\tilde{\psi}_{t} \varphi$.

Proof. By definition, we have $\operatorname{Ad}\left(\psi_{t}\right) \mathfrak{h}_{p}^{\infty}=\mathfrak{h}_{\psi_{l}(p)}^{\infty}$. Thus,

$$
\Phi\left(\mathfrak{h}_{\psi_{1}(p)}^{\infty}\right)=\Phi\left(\operatorname{Ad}\left(\psi_{t}\right) \mathfrak{h}_{p}^{\infty}\right)=\operatorname{Ad}\left(\tilde{\psi}_{t}\right)^{\prime} \mathfrak{h}_{\varphi(p)}^{\infty}=\mathfrak{h}_{\bar{\psi}_{1} \varphi(p)}^{\infty}
$$

This means $\varphi \psi_{t}=\tilde{\psi}_{t} \varphi$.

The above lemma shows that $\varphi$ maps a $C^{\infty}$ curve $(\exp t u)(p)$ onto a $C^{\infty}$ curve $(\exp t \Phi(u))(\varphi(p))$. Now recall how we made a $C^{\infty}$ local coordinate of a g-orbit (cf. Lemma 2.2). Then we have

THEOREM 5.1. Let $\Phi: \mathfrak{g} \rightarrow{ }^{\prime} g$ be an isomorphism which sends a nontrivial subcore $\mathfrak{h}$ to a subcore ' $\mathfrak{h}$. Then there is a homeomorphism $\varphi$ of $M-\Sigma_{0}(\mathfrak{h})$ onto $N-\Sigma_{0}(' \mathfrak{h})$ which is of $C^{\infty}$ on each g-orbit and satisfies $\Phi(u)=d \varphi u$.

CoRollary 5.1 (TheORem A). If, furthermore, $g$ and 'g satisfy condition $(\mathrm{C}, 5)$, then $\Sigma_{0}(\mathfrak{h})=\Sigma_{0}(' \mathfrak{h})=\varnothing$ and $\varphi: M \rightarrow N$ is a $C^{\infty}$ diffeomorphism such that $\Phi=d \varphi$. 


\section{Several remarks for former results.}

(a) Let $g$, ' $g$ be Lie algebras of $C^{\infty}$ vector fields with compact support on $M, N$, respectively. We assume that $\mathrm{g}$, 'g satisfy conditions (C,1), (C,2), (IC,2) and $(C, 4)$ in the introduction, $\Sigma_{0}(g)=\Sigma_{0}(' g)=\varnothing$, and, moreover, that these are modules over $C^{\infty}$ functions of $M$ and $N$, respectively. In this circumstance, the core of $g$ (resp. ' $g$ ) is just $g$ (resp. ' $g$ ).

Proposition 6.1. Let $\Phi: g \rightarrow$ 'g be an isomorphism. Then the homeomorphism $\varphi: M \rightarrow N$ obtained in Lemma 5.2 is a $C^{\infty}$ diffeomorphism.

Proof. For any $p \in M$, there is $u \in g$ such that $u(p) \neq 0$. Let $f$ be an arbitrary $C^{\infty}$ function on $M$. Then $f u-f(p) u \in \mathfrak{g}_{p}^{0}$ (the isotropy subalgebra); hence $(\Phi(f u)-f(p) \Phi(u))(\varphi(p))=0$ by Lemma 5.1. This means $\Phi(f u)$ $=\left(\varphi^{-1 *} f\right) \Phi(u)$ on some neighborhood of $\varphi(p)$. Since $\Phi(u)(\varphi(p)) \neq 0$ (Lemma 5.1), we see that $\varphi^{-1 *} f$ is of $C^{\infty}$; hence we have the desired result.

(b) Let $M$ be a connected, complete $C^{\infty}$ riemannian manifold. We denote by $\alpha$ a $C^{\infty}$ symplectic structure or a $C^{\infty}$ volume structure defined on $M$. In fact, $\alpha$ is a 2 or $n$ form on $M$, where $n=\operatorname{dim} M$. We define Lie algebras $\mathfrak{X}_{\alpha}(M)$ and $\mathfrak{X}_{\alpha, c}(M)$ as follows:

$$
\begin{aligned}
\mathfrak{X}_{\alpha}(M) & =\{u \in \mathfrak{X}(M) ; d(\alpha\lrcorner u)=0\} \quad \text { ( }\lrcorner \text { means the inner product.) } \\
\mathfrak{X}_{\alpha, c}(M) & =\{u \in \mathfrak{X}(M) ; \alpha\lrcorner u=d \gamma, \text { supp } \gamma \text { is compact }\},
\end{aligned}
$$

where $\mathfrak{X}(M)$ is the Lie algebra of all $C^{\infty}$ vector fields with compact support. It is well known that $\mathfrak{X}_{\alpha}(M)$ is a Lie algebra and the formula

$$
d(\alpha\lrcorner u\lrcorner v)=\alpha\lrcorner[u, v]
$$

shows that $\left[\mathfrak{X}_{\alpha}(M), \mathfrak{X}_{\alpha}(M)\right] \subset \mathfrak{X}_{\alpha, c}(M)$. As for the above formula, see [1, p. 98] for the case that $\alpha$ is a symplectic structure, but the similar computation shows that this is also true for volume structures.

LEMMA 6.1. $\mathfrak{X}_{\alpha, c}(M)$ is the core of $\mathfrak{X}_{\alpha}(M)$.

Proof. By a partition of unity, we see that $\mathfrak{X}_{\alpha, c}(M)$ has the property (P). Thus, $\mathfrak{X}_{\alpha, c}(M) \subset \mathcal{C}\left(\mathfrak{X}_{\alpha}(M)\right)$. For any $u \in \mathcal{C}\left(\mathfrak{X}_{\alpha}(M)\right)$, $u$ can be written as a finite sum $u=\sum_{\beta} u_{\beta}$ such that $u_{\beta} \in \mathcal{C}\left(\mathfrak{x}_{\alpha}(M)\right)$ and the diameter of the support of $u_{\beta}$ is sufficiently small. We may assume that supp $u_{\beta}$ is contained in an open neighborhood $U$ which is diffeomorphic to $\mathbf{R}^{n}$. Hence by the Poincare lemma, there is $\gamma_{\beta}$ such that $\left.\alpha\right\lrcorner u_{\beta}=d \gamma_{\beta}$ and supp $\gamma_{\beta} \subset U$, because $\left.d(\alpha\lrcorner u_{\beta}\right)$ $=0$. Therefore, $u_{\beta} \in \mathfrak{X}_{\alpha, c}(M)$ and hence we have $\mathfrak{X}_{\alpha, c}(M)=\mathcal{C}\left(\mathfrak{X}_{\alpha}(M)\right)$.

(c) Now, assume $M$ is a closed manifold. Then the core of $\mathfrak{x}_{\alpha}(M)$ is characterized algebraically by $\mathfrak{X}_{\alpha}(M)$. Namely, we have

Lemma 6.2. $\left[\mathfrak{X}_{\alpha}(M), \mathfrak{X}_{\alpha}(M)\right]=\mathfrak{X}_{\alpha, c}(M)$. 
Proof. Define a $C^{\infty}$ riemannian metric on $M$ such that the volume element $d \mu$ with respect to this metric is given by $\alpha$ itself (if $\alpha$ is a volume structure) or $\alpha^{m}$ (if $\alpha$ is a symplectic structure), where $2 m=n$. Since $M$ is closed, one can use the de Rham-Kodaira decomposition theorem, and as a result, if $\alpha\lrcorner u=d \gamma$, then $\gamma$ can be chosen so that it may satisfy $\gamma=\Delta \gamma^{\prime}$ for some $\gamma^{\prime}$ such that $\delta \gamma^{\prime}=0$, where $\Delta=d \delta+\delta d$. Namely, we have $\gamma=\delta d \gamma^{\prime}$.

Let $\left\{W_{\beta}\right\}$ be a finite open covering of $M$ such that every $W_{\beta}$ is contained in a coordinate neighborhood, and let $\left\{\phi_{\beta}\right\}$ be a partition of unity subordinate to $\left\{W_{\beta}\right\}$. We may assume that on every $W_{\beta}$ the structure $\alpha$ is written in the form $\alpha=d x_{1} \wedge \cdots \wedge d x_{n}$ or $\sum_{i=1}^{m} d x_{i} \wedge d x_{m+i}$. Set $\gamma=\sum_{\beta} \gamma_{\beta}$, where $\gamma_{\beta}$ $=\delta d\left(\phi_{\beta} \gamma^{\prime}\right)$. Using Stokes' theorem, we see that $\int_{W_{\beta}} \gamma_{\beta} d \mu=0$, where the integration means the following in the case that $\alpha$ is a volume structure: Using the local coordinate system $\left(x_{1}, \ldots, x_{n}\right)$, let $\left\{\omega_{p, q}\right\}$ be the dual basis of $d x_{1} \wedge \cdots \wedge \widehat{d x}_{p} \wedge \cdots \wedge \widehat{d x}_{q} \wedge \cdots \wedge d x_{n}$ in $\wedge^{n-2} T^{*} M$ with respect to the given riemannian metric on $M$ and set $\gamma_{\beta}=\sum_{p, q} \gamma_{p, q} \omega_{p, q}$. The integration means, of course, $\Sigma_{p, q}\left(\int_{W_{\beta}} \gamma_{p, q} d \mu\right) \omega_{p, q}$. In what follows in this section, the integration is always the componentwise integration with respect to the dual basis $\left\{\omega_{p, q}\right\}$.

In both cases, suitable extensions of $\partial / \partial x_{i}, i=1, \ldots, n$, are contained in $\mathfrak{X}_{\alpha}(M)$ (cf. [9, p. 121] for instance). We use the same notation for the extended vector fields. Note that we may assume without loss of generality that $W_{\beta}$ is a $2 \varepsilon$-cube in the coordinate expression.

Set

$$
I_{(1, \ldots, k)}=\int_{-\varepsilon}^{\varepsilon} \cdots \int_{-\varepsilon}^{\varepsilon} \gamma_{\beta}\left(t_{1}, \ldots, t_{k}, x_{k+1}, \ldots, x_{n}\right) d t_{1} \cdots d t_{k}
$$

and let $\phi(t)$ be a $C^{\infty}$ function of $(-\varepsilon, \varepsilon)$ such that supp $\phi \subset(-\varepsilon, \varepsilon)$ and $\int_{-\varepsilon}^{\varepsilon} \phi(t) d t=1$. Then we have

$$
\gamma_{\beta}=\gamma_{\beta, 1}+\gamma_{\beta, 2}+\cdots+\gamma_{\beta, n},
$$

where

$$
\begin{gathered}
\gamma_{\beta, 1}=\gamma_{\beta}-\phi\left(x_{1}\right) I_{(1)}, \\
\gamma_{\beta, k}=\phi\left(x_{1}\right) \cdots \phi\left(x_{k-1}\right)\left\{I_{(1,2, \ldots, k-1)}-\phi\left(x_{k}\right) I_{(1,2, \ldots, k)}\right\} \quad(1<k \leqslant n) .
\end{gathered}
$$

Since $I_{(1,2, \ldots, n)}=0$, we see $\gamma_{\beta, n}=\phi\left(x_{1}\right) \cdots \phi\left(x_{n-1}\right) I_{(1, \ldots, n-1)}$. Now, we see easily that $\int_{-\varepsilon}^{\varepsilon} \gamma_{\beta, k} d x_{k}=0$ for any $k$. Therefore $\tilde{\gamma}_{\beta, k}=\int_{-\varepsilon}^{x_{k}} \gamma_{\beta, k} d x_{k}$ is well defined and supp $\tilde{\gamma}_{\beta, k} \subset W_{\beta}$. Obviously, $\partial \tilde{\gamma}_{\beta, k} / \partial x_{k}=\gamma_{\beta, k}$. Let $u_{\beta, k}$ and $\tilde{u}_{\beta, k}$ be vector fields defined by $\left.\alpha\lrcorner u_{\beta, k}=d \gamma_{\beta, k}, \alpha\right\lrcorner \tilde{u}_{\beta, k}=d \tilde{\gamma}_{\beta, k}$. Then we have

$$
\left.\left.\left.\alpha\lrcorner u_{\beta, k}=\frac{\partial}{\partial x_{k}}(\alpha\lrcorner \tilde{u}_{\beta, k}\right)=\alpha\right\lrcorner\left(\frac{\partial}{\partial x_{k}} \tilde{u}_{\beta, k}\right)=\alpha\right\lrcorner\left[\frac{\partial}{\partial x_{k}}, \tilde{u}_{\beta, k}\right] .
$$


Hence, we see $u_{\beta, k} \in\left[\mathfrak{X}_{\alpha}(M), \mathfrak{X}_{\alpha}(M)\right]$. Thus, $\mathfrak{X}_{\alpha, c}(M) \subset\left[\mathfrak{X}_{\alpha}(M), \mathfrak{X}_{\alpha}(M)\right]$. This implies the equality, because the $\supset$ side is already known.

7. Multivalued primitive structures on each orbit of a core. Let $g$ be a Lie algebra of $C^{\infty}$ vector fields on $M$ with the conditions (C,1), (C,2), (IC,2) and $(\mathrm{C}, 4)$, and $N$ a $\mathcal{Q g})$-orbit of $p, p \in M-\Sigma_{0}(\mathcal{C}(\mathrm{g}))$, where $\mathcal{Q}(\mathrm{g})$ is the core of $\mathrm{g}$. The restriction $\mathcal{C}(\mathfrak{g})_{N}$ of $\mathcal{Q}(\mathrm{g})$ onto $N$ has the properties (i)-(iv) of Proposition 3.1. As a matter of course, $\mathcal{C}(\mathfrak{g})_{N}$ is infinitesimally transitive at every point $q \in N$. So, in this section we consider a Lie algebra $\Gamma$ of $C^{\infty}$ vector fields on a connected manifold $N$ which satisfies the following conditions:

(A) Every $u \in \Gamma$ is complete.

(B) $\operatorname{Ad}(\exp t u) \Gamma=\Gamma$ for any $u \in \Gamma, t \in \mathbf{R}$.

(C) $\int_{a}^{b} \operatorname{Ad}(\exp t u) v d t \in \Gamma$ for any $u, v \in \Gamma$.

(D) $\Gamma$ is infinitesimally transitive at every point.

(E) $\Gamma$ is primitive, i.e., the isotropy subalgebra $\Gamma_{p}$ at $p \in N$ is a maximal subalgebra of $\Gamma$ (cf. Theorem 3.1).

(F) Let $K, q$ be a compact set of $N$ and a point of $N$ such that $K \boxplus q$. Let $\varepsilon$ be an arbitrary positive number. For any $u \in \Gamma$ such that $u(q) \neq 0$, there is $v \in \Gamma$ with the following properties:

(a) $v \equiv u$ on a neighborhood of $q$.

(b) $v \equiv 0$ on a neighborhood of $K$.

(c) The diameter of the connected component of supp $v$ containing $q$ is less than $\varepsilon$, where the diameter is given by using an arbitrarily fixed complete $C^{\infty}$ riemannian metric on $N$.

Now, by condition (F) we see that $\operatorname{dim} \Gamma=\infty$. For any $p \in N$, we set $\Gamma_{p}^{k}=\left\{u \in \Gamma ; j_{p}^{k} u=0\right\}$, where $j_{p}^{k}$ is the $k$ th jet at $p$. We then have a series

$$
\Gamma=\Gamma^{-1} \supset \Gamma_{p}^{0} \supset \Gamma_{p}^{1} \supset \cdots \supset \Gamma_{p}^{k} \supset \cdots
$$

such that $\left[\Gamma_{p}^{k}, \Gamma_{p}^{l}\right] \subset \Gamma_{p}^{k+l}$ and $\operatorname{dim} \Gamma_{p}^{k} / \Gamma_{p}^{k+1}<\infty$. Let $\mathfrak{S}$ be the group generated by $\{\exp t u ; u \in \Gamma\}$. Then, $\subseteq$ acts transitively on $N$ because of condition (D) and the connectedness of $N$. By condition (B), we can define Ad $(\exp t u): \Gamma \rightarrow \Gamma$ as a Lie algebra automorphism, and it is easy to see that $\operatorname{Ad}(\exp t u) \Gamma_{p}^{k}=\Gamma_{\varphi_{t}(p)}^{k}$, where $\varphi_{t}=\exp t u$.

LEMMA 7.1. Let $\Gamma_{p}^{\infty}=\cap \Gamma_{p}^{k}$. Then $\Gamma / \Gamma_{p}^{\infty}$ is an infinite dimensional filtered Lie algebra with the filtration $\left\{\Gamma_{p}^{k} / \Gamma_{p}^{\infty}\right\}$.

Proof. It is clear that $\left\{\Gamma_{p}^{k} / \Gamma_{p}^{\infty}, k=-1,0,1,2, \ldots\right\}$ is a filtered Lie algebra (cf. [7], for instance, for the definition of filtered Lie algebra). Assume $\Gamma / \Gamma_{p}^{\infty}$ is a finite dimensional Lie algebra. Then, of course, $\Gamma_{p}^{\infty}$ is a closed finite codimensional ideal of $\Gamma$. By Lemma 2.1, we have $\operatorname{Ad}^{p}(\exp t u) \Gamma_{p}^{\infty}=\Gamma_{p}^{\infty}$ for any $u \in \Gamma$. On the other hand, we have $\operatorname{Ad}(\exp t u) \Gamma_{p}^{\infty}=\Gamma_{\varphi_{1}(p)}^{\infty}$ by definition. Hence $\Gamma_{p}^{\infty}=\Gamma_{q}^{\infty}$ for any $q \in N$. This means $\Gamma_{p}^{\infty}=\{0\}$; hence $\operatorname{dim} \Gamma<\infty$. 
Now, by taking $\left\{\Gamma_{p}^{k} / \Gamma_{p}^{\infty}, k=-1,0,1,2, \ldots\right\}$ as a basis of neighborhoods of zero, we define a topology on that filtered Lie algebra. Let $\mathfrak{U}^{k}(\Gamma, p)$ be the completion of $\Gamma_{p}^{k} / \Gamma_{p}^{\infty}$ in the above topology. We set $\mathfrak{A}^{-1}(\Gamma, p)=\mathfrak{A}(\Gamma, p)$. We have then a series $\mathfrak{A}(\Gamma, p) \supset \mathfrak{A}^{0}(\Gamma, p) \supset \cdots \supset \mathfrak{A}^{k}(\Gamma, p) \supset \cdots$ such that $\cap_{k} \mathfrak{A}^{k}(\Gamma, p)=\{0\},\left[\mathfrak{A}^{k}(\Gamma, p), \mathfrak{A}^{l}(\Gamma, p)\right] \subset \mathfrak{A}^{k+l}(\Gamma, p), \mathfrak{A}^{k}(\Gamma, p) / \mathfrak{A}^{k+1}(\Gamma, p)$ $=\Gamma_{p}^{k} / \Gamma_{p}^{k+1}$. Of course, $\mathfrak{A}(\Gamma, p)$ is complete in the above topology. Now, by condition (E) we see that $\left\{\mathfrak{U}^{k}(\Gamma, p), k=-1,0,1,2, \ldots\right\}$ is a complete, primitive transitive filtered Lie algebra, that is, primitive TLA in the notation of [7].

Let $F$ be the totality of formal power series $\sum a_{\alpha}^{i} x^{\alpha} \partial / \partial x_{i}$ of vector fields on $\mathbf{R}^{n}$. Let $F^{k}=\left\{u \in F ; u=\sum_{|\alpha|>k+1} a_{\alpha}^{i} x^{\alpha} \partial / \partial x_{i}\right\}$. Define a topology by taking $\left\{F^{k}\right\}$ as a basis of neighborhoods of 0 . Then $F$ is a complete filtered Lie algebra. Remark that by the same procedure as above we can make a complete filtered Lie algebra $\mathfrak{U}\left(\boldsymbol{\Gamma}^{\prime}, 0\right) \subset \boldsymbol{F}$ for any (local) Lie algebra $\Gamma^{\prime}$ defined on a neighborhood of the origin of $\mathbf{R}^{n}$. So for that given $\Gamma$, we can make a local Lie algebra $\Gamma^{\prime}$ (using local coordinates at $p$ ) such that $\mathfrak{A}\left(\Gamma^{\prime}, 0\right)$ is isomorphic to $\mathfrak{A}(\Gamma, p)$. We fix such Lie algebra $\Gamma^{\prime}$ on a neighborhood of 0 of $\mathbf{R}^{n}$.

Let $G_{\Gamma}$ be the linear group of all jets $j_{0}^{1} f$ of a local diffeomorphism $f:\left(\mathbf{R}^{n}, 0\right) \rightarrow\left(\mathbf{R}^{n}, 0\right)$ such that $f$ induces an isomorphism of $\mathfrak{A}\left(\Gamma^{\prime}, 0\right)$ onto itself. Let $B_{q}$ be the space of all jets $j_{0}^{1} h$ of a local diffeomorphism $h:\left(\mathbf{R}^{n}, 0\right)$ $\rightarrow(N, q)$ such that $h$ induces an isomorphism of $\mathfrak{A}\left(\Gamma^{\prime}, 0\right)$ onto $\mathfrak{A}(\Gamma, q)$. Remark that $\operatorname{Ad}(\exp t u) \mathfrak{A}^{k}(\Gamma, p)=\mathfrak{A}^{k}\left(\Gamma, \varphi_{t}(p)\right), \varphi_{t}=\exp t u$, for any $k$, and therefore $d \varphi_{t} B_{p}=B_{\varphi_{1}(p)}$. Namely, the bundle $B=\cup_{p \in N} B_{p}$ is invariant under the

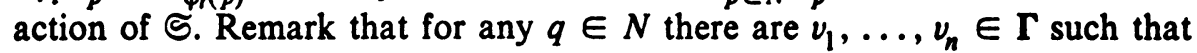
the mapping $\left(t_{1}, \ldots, t_{n}\right) \rightarrow\left(\exp \left(t_{1} v_{1}+\cdots+t_{n} v_{n}\right)\right)(q)$ gives a $C^{\infty}$ coordinate at $q$ (cf. Lemma 2.2). Set $\varphi_{\left(t_{1}, \ldots, t_{n}\right)}=\exp \left(t_{1} v_{1}+\cdots+t_{n} v_{n}\right)$. Since $d \varphi_{\left(t_{1}, \ldots, t_{n}\right)} B_{p}=B_{\left.\varphi_{\left(t_{1}\right.} \ldots, t_{0}\right)}(p)$, this gives a $C^{\infty}$ local trivialization of $B$ and hence $B$ is a $C^{\infty}$ bundle over $M$. It is easy to see that $B$ is a $G_{\Gamma}$-principal bundle, which is canonically imbedded in the frame bundle, i.e., $B$ is a $G_{\Gamma}$-structure. The following is clear by the above arguments:

LEMMA 7.2. The group $\subseteq$ is a subgroup of the group of the automorphisms of the $G_{\Gamma}$-structure defined above.

Let $g_{\Gamma}$ be the Lie algebra of $G_{\Gamma} \cdot g_{\Gamma}$ is a subalgebra of $g l(n)(=n$-by- $n$ matrices). Since $\operatorname{dim} \Gamma=\infty, g_{\Gamma}$ must be of infinite type.

LEMMA 7.3. $\mathrm{g}_{\Gamma}$ contains an element of rank 1.

Proof. Suppose $g_{\Gamma}$ contains no element of rank 1 . Then it is well known that there is a linear, elliptic differential operator $D$ of order 2 on $N$ such that any infinitesimal $G_{\Gamma^{-}}$-automorphism $u$ on $N$ satisfies $D u=0$ (cf. for instance [8]). Now, the following is also well known (cf. [6, Lemma 3.11 and its proof, pp. 196-197]). For every $s \geqslant 0$, there exists $\delta(s)>0$ depending on $s$ such that if the diameter of the support of a $C^{\infty}$ vector field $u$ is less than $\delta(s)$, then 
$\|D u\|_{s} \geqslant C_{0}\|u\|_{s+2}$, where the norms are usual $L_{2}^{s}$ - and $L_{2}^{s+2}$-norms.

Since $D u=0$ for any $u \in \Gamma$, the above inequality contradicts condition (F). Hence, we have that $g_{\Gamma}$ contains an element of rank one.

Now fortunately the primitive TLA's are completely classified (cf. [12], [7], [3] and [11]). There are 14 cases of such primitive TLA's. In the list of primitive TLA's, we can pick up the primitive TLA's whose $g_{\Gamma}$ contains elements of rank one. Then, we have the following six cases:

(i) $\mathfrak{H}\left(\mathfrak{X}\left(\mathbf{R}^{n}\right), 0\right): \mathfrak{X}\left(\mathbf{R}^{n}\right)$ is the Lie algebra of all $C^{\infty}$ vector fields on $\mathbf{R}^{n}$.

(ii) $\mathfrak{A}\left(\mathfrak{X}_{\omega}\left(\mathbf{R}^{n}\right), 0\right): n=2 m+1$ and $\omega=d x_{0}+\sum_{i=1}^{m} x_{m+i} d x_{i}, \mathfrak{X}_{\omega}\left(\mathbf{R}^{n}\right)$ is the Lie algebra of all $C^{\infty}$ contact transformations.

(iii) and (iv) $\mathfrak{U}\left(\mathfrak{X}_{\alpha}\left(\mathbf{R}^{n}\right), 0\right): \alpha=d x_{1} \wedge \cdots \wedge d x_{n}$ or $\sum d x_{i} \wedge d x_{m+i}$ $(n=2 m) . \mathfrak{X}_{\alpha}\left(\mathbf{R}^{n}\right)$ is the Lie algebra of all infinitesimal $\alpha$-preserving transformations on $\mathbf{R}^{n}$.

(v) and (vi) $\mathfrak{A}\left(\mathfrak{X}_{\alpha, \text { conf }}\left(\mathbf{R}^{n}\right), 0\right): \alpha$ is as above, $\mathfrak{X}_{\alpha, \text { conf }}\left(\mathbf{R}^{n}\right)=\left\{u \in \mathfrak{X}\left(\mathbf{R}^{n}\right)\right.$; $d(\alpha\lrcorner u)=c \alpha\}$, where $c$ is a constant depending on $u$.

All others are related to complex structures and it is not hard to see that $g_{\Gamma}$ contains no rank one element because $g_{\Gamma}$ is contained in

$$
\mathrm{C} \cdot \text { id }+\mathfrak{A}^{0}\left(\Gamma^{\prime}, 0\right) / \mathfrak{A}^{1}\left(\Gamma^{\prime}, 0\right) \text {. }
$$

From now on, the argument goes case by case.

(a) The case $\mathfrak{A}(\Gamma, p) \cong \mathfrak{A}\left(\mathfrak{X}\left(\mathbf{R}^{n}\right), 0\right)$.

If this is the case, we have nothing to prove. $N$ has a $C^{\infty}$ structure. However, we have to remark that the above isomorphism means the following: Let $x_{1}, \ldots, x_{n}$ be a local coordinate at $p$. Take the Taylor expansions of all $u \in \Gamma$ at $p$ with respect to that coordinate and take the closure in $F$. Then, we get a Lie subalgebra $\mathfrak{A}\left(\Gamma,\left(x_{1}, \ldots, x_{n}\right), p\right)$ of $F$, but the above isomorphism means that $\mathfrak{U}\left(\Gamma,\left(x_{1}, \ldots, x_{n}\right), p\right)=\mathfrak{U}\left(\mathfrak{X}\left(\mathbf{R}^{n}\right), 0\right)$ for a suitable choice of coordinate.

(b) The case $\mathfrak{A}(\Gamma, p) \cong \mathfrak{A}\left(\mathfrak{X}_{\omega}\left(\mathbf{R}^{n}\right), 0\right)$.

For any point $p \in N$, there is a local coordinate $x_{0}, x_{1}, \ldots, x_{2 m}$ at $p$ such that $\mathfrak{A}\left(\Gamma,\left(x_{0}, x_{1}, \ldots, x_{2 m}\right), p\right)=\mathfrak{U}\left(\mathfrak{X}_{\omega}\left(\mathbf{R}^{n}\right), 0\right)$ where $\omega=d x_{0}$ $+\sum_{i=1}^{m} x_{m+i} d x_{i}$. Remark that the space $\left.E=\left\{X \in T_{0} \mathbf{R}^{n} ; \omega\right\lrcorner X=0\right\}$ is characterized as the invariant subspace of the linear isotropy subalgebra $\mathfrak{U}^{0}\left(\mathfrak{X}_{\omega}\left(\mathbf{R}^{n}\right), 0\right) / \mathfrak{U}^{1}\left(\mathfrak{X}_{\omega}\left(\mathbf{R}^{n}\right), 0\right)$ of $\mathfrak{X}_{\omega}\left(\mathbf{R}^{n}\right)$. So using the above local coordinate we define a one-dimensional subspace $L_{p}=\left\{\lambda d x_{0} ; \lambda \in \mathbf{R}\right\}$ in the cotangent space $T_{p}^{*} N$ at $p$. Since $\operatorname{Ad}(\exp t u) \mathfrak{U}(\Gamma, p)=\mathfrak{A}\left(\Gamma, \varphi_{t}(p)\right)$, we see that $\varphi_{l}^{*} L_{p}$ $=L_{\varphi_{t}^{-1}(p)}$, and by the same reasoning as to give a $C^{\infty}$ structure on $B$, we see that $L=\bigcup_{p \in N} L_{p}$ is a $C^{\infty}$ line bundle over $N$. Hence, there is at most a twovalued $C^{\infty}$ cross-section $\omega$ of $L-\{0\}$. By the same argument as in [9, pp. 121 and 134], we see that $\omega$ is locally a contact 1 -form. We call $\omega$ a multivalued contact structure on $N$, but this is not good terminology. The automorphism group of this structure is the group of diffeomorphisms $\varphi$ which leave $L$ invariant. Of course, $\mathfrak{S}$ is its subgroup. 
(c) The case $\mathfrak{U}(\Gamma, p) \cong \mathfrak{U}\left(\mathfrak{X}_{\alpha}\left(\mathbf{R}^{n}\right), 0\right), \alpha=d x_{1} \wedge \cdots \wedge d x_{n}$ or $\Sigma d x_{i}$
$\wedge d x_{m+i}$.

For every point $p \in N$, there is a local coordinate $x_{1}, \ldots, x_{n}$ such that $\mathfrak{U}\left(\Gamma,\left(x_{1}, \ldots, x_{n}\right), p\right)=\mathfrak{A}\left(\mathfrak{X}_{\alpha}\left(\mathbf{R}^{n}\right), 0\right)$. Remark that $\alpha$ can be characterized by $\mathfrak{A}\left(\mathfrak{X}_{\alpha}\left(\mathbf{R}^{n}\right), 0\right)$ up to a constant factor. (See [9, pp. 133-134] for the precise reasoning.) Using the above local coordinate, we define a line bundle $L=\cup_{p \in N} L_{p}, L_{p}=\mathbf{R} \cdot d x_{1} \wedge \cdots \wedge d x_{n}$ or $\mathbf{R} \cdot\left(\sum d x_{i} \wedge d x_{m .}\right)$. By the same reasoning as in (b), $L$ is a $C^{\infty}$ line bundle over $N$ and the group $\subseteq$ leaves $L$ invariant. Let $\pi: L \rightarrow N$ be the projection. Remark that any local section of $L-\{0\}$ is of maximal rank.

Now, every $u \in \Gamma$ defines a $C^{\infty}$ vector field $\hat{u}$ on $L$. Indeed, for any $\delta \in L$, we set $\hat{u}(\delta)=d /\left.d t\right|_{t=0}(\exp -t u)^{*} \delta$. (Note that $c(t)=(\exp -t u)^{*} \delta$ is a $C^{\infty}$ curve in $L$ such that $c(0)=\delta$.) We set $\hat{\Gamma}(\delta)=\{\hat{u}(\delta) ; u \in \Gamma\}$.

LEMMA 7.4. $D=\cup_{\delta \in L} \hat{\mathbf{f}}(\delta)$ is an involutive horizontal distribution on $L$, where "horizontal" means that $(d \pi)_{\delta}: D_{\delta} \rightarrow T_{\pi \delta} N$ is an isomorphism for any $\delta \in L$.

Proof. Let $\delta \in L_{p}$. We use the local coordinate $x_{1}, \ldots, x_{n}$ at $p$. $\delta$ is written as $\delta=\lambda d x_{1} \wedge \cdots \wedge d x_{n}$ or $\lambda\left(\sum d x_{i} \wedge d x_{m+i}\right)$, where $\lambda \in \mathbf{R} . \hat{u}(\delta)$ is then given by $\left(-\sum u^{i}(p) \partial / \partial x_{i},\left(\mathfrak{\Sigma}_{u} \bar{\delta}\right)(p)\right)$, where $u=\sum u^{i} \partial / \partial x_{i}$ and $\delta$ is just $\delta$ regarded as a constant tensor field on that coordinate system. Hence, we see immediately that $\hat{\Gamma}(\delta)$ contains no vertical vector, because $\left(\mathfrak{L}_{u} \bar{\delta}\right)(p)=\mathfrak{R}_{u} \lambda \alpha$ $=0$. Hence, we have $D$ is a horizontal distribution on $L$. This is involutive because $\Gamma$ is a Lie algebra.

Now we take a nontrivial maximal integral submanifold of this distribution $D$ and call it $\alpha_{0}$. It is clear that $\alpha_{0}$ is invariant under the action of $\mathcal{S}$, and, hence, locally $\mathfrak{R}_{u} \alpha_{0}=0$ for any $u \in \Gamma$. Since $\Gamma$ is infinitesimally transitive at every point, we see that $\alpha_{0}$ is locally constant and hence a closed form. Since $\alpha_{0}$ is of maximal rank, we see that $\alpha_{0}$ is locally a volume form or a symplectic form.

We call $\alpha_{0}$ a multivalued symplectic or volume structure on $N$. For each $p \in N$, there is a local coordinate $x_{1}, \ldots, x_{n}$ such that $\alpha_{0}=\left\{\lambda d x_{1} \wedge \cdots\right.$ $\left.\wedge d x_{n}\right\}$ or $\left\{\lambda\left(\sum d x_{i} \wedge d x_{m+i}\right)\right\}$, where $\lambda$ moves in a subset $\Lambda$ of $\mathbf{R}-\{0\}$. Let $\tilde{N}$ be the universal covering space of $N$ and $\Pi$ the covering transformation group. Then the multivalued structure on $N$ is nothing but a symplectic or volume structure $\tilde{\alpha}_{0}$ on $\tilde{N}$ with $\psi^{*} \tilde{\alpha}_{0}=\tau(\psi) \tilde{\alpha}_{0}$ for $\psi \in \Pi$, where $\tau(\psi)$ is the constant depending on $\psi$. The set $\Lambda$ is given in fact by $\tau(\Pi)$; hence $\Lambda$ is a multiplicative subgroup of $\mathbf{R}-\{0\}$.

A typical example of multivalued structure is given as follows: Let $N=\mathbf{R}^{2} / \mathbf{Z}^{2}$. On $\mathbf{R}^{2}$, we consider $\tilde{\alpha}_{0}=e^{a x+b y} d x \wedge d y$. Then, this defines a multivalued structure $\alpha_{0}$ on the torus $\mathbf{R}^{2} / \mathbf{Z}^{2}$.

(d) The case $\mathfrak{A}(\Gamma, p) \cong \mathfrak{U}\left(\mathfrak{X}_{\alpha, \text { conf }}\left(\mathbf{R}^{n}\right), 0\right)$. 
Remark that

$$
\mathfrak{U}\left(\mathfrak{X}_{\alpha, \text { conf }}\left(\mathbf{R}^{n}\right), 0\right)=\mathbf{R}\left(\sum x_{i} \frac{\partial}{\partial x_{i}}\right) \oplus \mathfrak{U}\left(\mathfrak{X}_{\alpha}\left(\mathbf{R}^{n}\right), 0\right) .
$$

It is not hard to see that $\mathfrak{U}\left(\mathfrak{X}_{\alpha}\left(\mathbf{R}^{n}\right), 0\right)$ is the only one closed ideal of codimension one which is transitive and primitive by itself. (Here, we used the classification table.) Now, at each $p \in M$, there is one codimensional ideal $\Gamma_{p}^{*}$ of $\Gamma$ such that $\mathfrak{U}\left(\Gamma_{p}^{*}, p\right) \cong \mathfrak{U}\left(\mathfrak{X}_{\alpha}\left(\mathbf{R}^{n}\right), 0\right)$ and this isomorphism can be extended to an isomorphism of $\mathfrak{U}(\Gamma, p)$ onto $\mathfrak{U}\left(\mathfrak{X}_{\alpha, \text { conf }}\left(\mathbf{R}^{n}\right), 0\right)$. It is now clear that $\operatorname{Ad}(\exp t u) \Gamma_{p}^{*}=\Gamma_{\varphi_{1}(p)}^{*}$. However, by Lemma 2.1 , we see that $\Gamma_{p}^{*}=\Gamma_{\varphi_{1}(p)}^{*}$; hence $\Gamma_{p}^{*}$ does not depend on $p$. Thus, we write $\Gamma^{*}$ instead of $\Gamma_{p}^{*}$.

Using $\Gamma^{*}$, we make a line bundle $L$ and an involutive horizontal distribution $D$ on $L$ by the same procedure as in (c). Since $\operatorname{Ad}(\exp t u) \Gamma^{*}=\Gamma^{*}$ for any $u \in \Gamma$, we see that $D$ is invariant under the natural action of the group $\mathbb{S}$. We have already seen that a nontrivial maximal integral submanifold $\alpha_{0}$ of $D$ is a multivalued volume or symplectic structure.

Note that for any $v \in \Gamma,(\exp t v)^{*} \alpha_{0}$ is also an integral submanifold of $D$ and hence $\mathfrak{L}_{u}(\exp t v)^{*} \alpha_{0}=0$ locally for any $u \in \Gamma^{*}$, but this shows $(\exp t v)^{*} \alpha_{0}=c \alpha_{0}$, because $\Gamma^{*}$ itself is infinitesimally transitive at every point.

Now, we recall condition (F). Then, it is clear that $c$ must be 1 . In other words, $\Gamma^{*}=\Gamma$. Hence, the case $\mathfrak{U}(\Gamma, p) \cong \mathfrak{U}\left(\mathfrak{X}_{\alpha, \text { conf }}\left(\mathbf{R}^{n}\right), 0\right)$ does not take place.

By summing up the above results, we have

THEOREM 7.1. There is a multivalued primitive structure $\alpha_{0}$ on $N$ such that the group $\subseteq$ is contained in the automorphism group of $\alpha_{0}$ structure, where $\alpha_{0}$ is, of course, one of a $C^{\infty}$ structure on $N\left(\alpha_{0}=\varnothing\right)$, an at most two-valued contact form $\left(\alpha_{0}=\omega\right)$, a multivalued symplectic form $\left(\alpha_{0}=\Omega\right)$ and a multivalued volume form $\left(\alpha_{0}=d V\right)$.

8. Classification of transitive cores, proof of Theorem B. Let $g$ be a Lie algebra of $C^{\infty}$ vector fields on $M$ with $(C, 1)-(C, 5)$, and $\Subset$ the core of $g$. In this section, we assume furthermore that $\mathbb{C}$ itself is infinitesimally transitive at every point. By Theorem 7.1, there is a multivalued primitive structure $\alpha_{0}$ on $M$ such that $\mathfrak{E}$ is a subalgebra of the Lie algebra of all infinitesimal

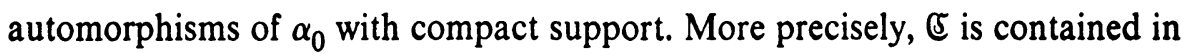
one of the following Lie algebras:

(i) $\mathfrak{X}(M)$ : Lie algebra of all vector fields with compact support.

(ii) $\mathfrak{X}_{\omega}(M)=\left\{u \in \mathfrak{X}(M)\right.$; $\left.(\exp t u)^{*} L=L\right\}$, where $L$ is the line bundle given in $\$ 7(\mathrm{~b})$.

(iii) $\mathfrak{X}_{\Omega}(M)=\left\{u \in \mathfrak{X}(M) ; \mathfrak{I}_{u} \Omega=0\right\}$ where $\Omega$ is a multivalued symplectic structure on $M$.

(iv) $\mathfrak{X}_{d V}(M)=\left\{u \in \mathfrak{X}(M) ; \mathfrak{I}_{u} d V=0\right\}$, where $d V$ is a multivalued volume structure on $M$. 
Since

$$
\begin{aligned}
\Omega & =\left\{\lambda\left(\sum d x_{i} \wedge d x_{i+m}\right): \lambda \in \Lambda\right\}, \\
d V & =\left\{\lambda d x_{1} \wedge \cdots \wedge d x_{n} ; \lambda \in \Lambda^{\prime}\right\}
\end{aligned}
$$

and $\Lambda, \Lambda^{\prime} \subset \mathbf{R}$ on a suitably chosen local coordinate neighborhood at every point, we see that the definitions (iii) and (iv) make sense.

Let $\alpha_{0}$ be one of $\varnothing$ (i.e., the case (i)), $\omega, \Omega$ and $d V$ and let $\alpha$ be one of $\varnothing$, $d x_{0}+\sum_{i=1}^{m} x_{i} d x_{i+m}(n=2 m+1), \sum d x_{i} \wedge d x_{m+i}(n=2 m)$ and $d x_{1} \wedge \cdots$ $\wedge d x_{n}$ on $\mathbf{R}^{n} . \mathfrak{X}_{\alpha}\left(\mathbf{R}^{n}\right)$ is the Lie algebra defined just after Lemma 7.3. Now the following is easy to see (see for instance $[9, \S \mathrm{X}, \mathrm{p} .119])$ :

LeMMA 8.1.

$$
\mathfrak{U}\left(\mathfrak{X}_{\alpha_{0}}(M), p\right) \cong \mathfrak{A}\left(\mathfrak{X}_{\alpha}\left(\mathbf{R}^{n}\right), 0\right) \text { for any } p \in M,
$$

where $\mathfrak{A}\left(\mathfrak{X}_{\alpha_{0}}(M), p\right)$ is the Lie algebra defined between Lemmas 7.1 and 7.2.

The following lemma is proved by using property $(C, 3)$.

LEMMA 8.2. g $\supset \mathfrak{X}_{\alpha_{0}}(M) \supset \mathfrak{C}$.

Proof. Recall the argument in the previous section. By the arguments in (a), (b), (c) in $\$ 7$ and Lemma 8.1, we get that $\mathfrak{U}(\mathbb{E}, p)=\mathfrak{U}\left(\mathfrak{X}_{\alpha_{0}}(M), p\right)$ for every $p \in M$. This equality means the following: Let $u \in \mathfrak{X}_{\alpha_{\alpha}}(M)$. For any $p$, and for any $k \geqslant 0$, there is $v_{p}^{k} \in \mathbb{C}$ such that $j_{p}^{k} u=j_{p}^{k} v_{p}^{k}$. Thus we see that $\mathfrak{g} \supset \mathfrak{X}_{\alpha_{0}}(M)$ because of property $(C, 3)$. The inclusion $\mathbb{E} \subset \mathfrak{X}_{\alpha_{0}}(M)$ is already proved in $\$ 7$.

Corollary. $\mathbb{E}$ is equal to the core of $\mathfrak{X}_{\alpha_{0}}(M)$.

The proof is trivial because the core is the maximal subspace with property (P).

Therefore, for the proof of Theorem B, we have only to fix the core of $\mathfrak{X}_{\alpha_{0}}(M)$.

(a) $\alpha_{0}=\varnothing$. There is nothing to prove. We see that the core of $\mathfrak{X}(M)$ is $\mathfrak{X}(M)$ itself.

(b) $\alpha_{0}=\omega$.

LEMMA 8.3. The core of $\mathfrak{X}_{\omega}(M)$ is $\mathfrak{X}_{\omega}(M)$ itself.

Proof. In case $\omega$ is a single-valued form, it is easily seen by the following: If $\mathfrak{R}_{u} \omega=h \omega$, then for a given $f \in C^{\infty}(M)$ there exists a $C^{\infty}$-vector field $v$ with supp $v \subset \operatorname{supp} f$ such that if $u^{\prime}=f u+v$, then $\mathfrak{R}_{u^{\prime}} \omega=h^{\prime} \omega$. Also, $v$ is unique if $\omega\lrcorner v=0$. (See $[9$, Lemma 8.3.1, p. 98] for a precise proof.) Now assume that the line bundle $L$ defined in $\S 7$ (b) is not a trivial bundle. Then we take a double covering $M_{2}$ of $M$ so that the pull-back of $L$ may become a 
trivial bundle on $M_{2}$. Let $\pi: M_{2} \rightarrow M$ be the projection and $\psi$ the covering transformation such that $\psi^{2}=$ id . We set $\bar{\omega}=\pi^{*} \omega$. Then $\bar{\omega}$ is a single-valued contact form on $M_{2}$. We denote by $\bar{u}$ the lift of a vector field $u$ on $M$.

For any $u \in \mathfrak{X}_{\omega}(M)$ and for a sufficiently small $\varepsilon, \bar{u}$ can be written as a finite sum $\bar{u}=\sum_{\beta} v_{\beta}$ such that $v_{\beta} \in \mathfrak{X}_{\bar{\omega}}\left(M_{2}\right)$ and diam $\left(\operatorname{supp} v_{\beta}\right)<\varepsilon$. Put $\bar{u}_{\beta}=\frac{1}{2}\left(v_{\beta}+d \psi v_{\beta}\right)$. Then, we see that $\bar{u}_{\beta}$ is the lift of a vector field $u_{\beta}$ on $M$. Obviously, diam $\left(\operatorname{supp} u_{\beta}\right)<\varepsilon$, because $\psi$ is an isometry. Since $\psi$ is also a contact transformation on $M_{2}$ (i.e., $\psi^{*} \bar{\omega}=f \bar{\omega}$ ), we have that $\bar{u}_{\beta} \in \mathfrak{X}_{\bar{\alpha}}\left(M_{2}\right)$, but this means that $u_{\beta} \in \mathfrak{X}_{\omega}(M)$. Since $\bar{u}=\sum_{\beta} \bar{u}_{\beta}$, we see that $u=\Sigma_{\beta} u_{\beta}$. Our assertion is thereby proved.

(c) $\alpha_{0}=\Omega$ or $d V$.

Let $L$ be the line bundle defined in $\S 7(\mathrm{c}) . \alpha_{0}$ is a multivalued section of $L$. If $\alpha_{0}$ is a single-valued section, then the core of $\mathfrak{X}_{\alpha_{0}}(M)$ is already fixed by Lemma 6.1. If $\alpha_{0}$ is multivalued, the exact determination of the core will be given in the next section. Here, we will make a preparation for that.

Assume the line bundle $L$ is not a trivial bundle. We take a double covering $M_{2}$ so that the pull-back $\bar{L}$ of $L$ may become a trivial bundle over $M_{2}$. Let $\bar{\alpha}_{0}$ be the pull-back of $\alpha_{0}$. $\bar{\alpha}_{0}$ may still be a multivalued form on $M_{2}$. Let $\psi$ be the covering transformation such that $\psi^{2}=\mathrm{id}, \psi \neq \mathrm{id}$. Then we have $\psi^{*} \alpha_{0}$ $=-\alpha_{0}$. Let $\bar{u}$ be the lift of a vector field $u$ on $M$ onto $M_{2}$. Let $\mathcal{C}\left(\mathcal{X}_{\alpha_{0}}\left(M_{2}\right)\right)$ be the core of $\mathfrak{X}_{\bar{\alpha}_{0}}\left(M_{2}\right)$.

LEMma 8.4. The lift of the core of $\mathfrak{X}_{\alpha_{0}}(M)$ onto $M_{2}$ is given by $\{w$ $\left.\in \mathcal{C}\left(\mathfrak{X}_{\bar{\alpha}_{0}}\left(M_{2}\right)\right) ; d \psi w=w\right\}$.

Proof. Let $\varrho\left(\mathfrak{X}_{\alpha_{0}}(M)\right), \mathcal{C}\left(\mathfrak{X}_{\alpha_{0}}(M)\right)^{-}$be the core of $\mathfrak{X}_{\alpha_{0}}(M)$ and its lift, respectively. For any $w \in \mathcal{C}\left(\mathfrak{X}_{\alpha_{0}}\left(M_{2}\right)\right)$ such that $d \psi w=w$, it is clear that $w \in \mathcal{C}\left(\mathfrak{X}_{\alpha_{0}}(M)\right)^{-}$by the same reasoning as in Lemma 8.3. Hence $\mathcal{C}\left(\mathfrak{X}_{\alpha_{0}}(M)\right)^{-}$ $\supset\left\{w \in \mathcal{C}\left(\mathfrak{x}_{a_{0}}\left(M_{2}\right)\right) ; d \psi w=w\right\}$.

On the other hand, for any sufficiently small $\varepsilon>0$, every $u \in \mathcal{C}\left(\mathfrak{X}_{\alpha_{0}}(M)\right)$ can be written as a finite sum $u=\sum_{\beta} u_{\beta}$ such that $u_{\beta} \in \mathcal{C}\left(\mathfrak{X}_{\alpha_{0}}(M)\right)$ and $\operatorname{diam}\left(\operatorname{supp} u_{\beta}\right)<\varepsilon$. Therefore, $\bar{u}_{\beta} \in \mathcal{C}\left(\mathfrak{X}_{\alpha_{0}}(M)\right)^{-}$and $\bar{u}_{\beta}=v_{\beta}+d \psi v_{\beta}$ such that diam $\left(\operatorname{supp} v_{\beta}\right)<\varepsilon$. We consider a linear subspace of $\mathfrak{X}_{\bar{\alpha}_{0}}\left(M_{2}\right)$ spanned by those $v_{\beta}, d \psi v_{\beta}$ for every $u \in \mathfrak{X}_{\alpha_{0}}(M)$. Then it is easy to see that the linear space has property $(\mathrm{P})$. Hence, we have $\mathcal{C}\left(\mathfrak{X}_{\alpha_{0}}(M)\right)^{-} \subset\left\{w \in \mathcal{C}\left(\mathfrak{X}_{\alpha_{0}}\left(M_{2}\right)\right)\right.$; $d \psi w=w\}$. Our assertion is thereby proved.

REMARK. By the above lemma, we have only to treat the case that $L$ is trivial to know the core of $\mathfrak{X}_{\alpha_{0}}(M)$.

9. Classification of transitive cores. II.

(A) Now we start with a multivalued symplectic or volume structure $\alpha_{0}$ on $M$, which is given as a multivalued section of the trivial line bundle $L$. Since $L$ is trivial, there is a $C^{\infty}$ section $\beta$ of $L-\{0\}$. Therefore, we know that $M$ is orientable. Let $\tilde{M}$ be the universal covering space of $M$ and $\tilde{\alpha}_{0}, \tilde{\beta}$ the pull- 
backs of $\alpha_{0}, \beta$, respectively. $\tilde{\alpha}_{0}$ is then a single-valued symplectic or volume form on $\tilde{M}$. We have then $\tilde{\beta}=g \tilde{\alpha}_{0}$ on $\tilde{M}$, where we may assume that $g$ is a positive $C^{\infty}$ function. If $\alpha_{0}$ is a single-valued form on $M$, then we set $g \equiv 1$. Let $\Pi$ be the covering transformation group of $\tilde{M}$. For any $\psi \in \Pi$, we see $\psi^{*} \tilde{\beta}=\tilde{\beta}$, and $\psi^{*} \tilde{\alpha}_{0}=\tau(\psi) \tilde{\alpha}_{0}$ where $\tau(\psi)$ is a constant depending on $\psi$ (cf. the last paragraph of $\$ 7)$. Therefore, we see $\psi^{*} g=g / \tau(\psi)$. Though $g$ is not a function on $M, \tilde{\theta}=d g / g$ is a closed 1-form such that $\psi^{*} \tilde{\theta}=\tilde{\theta}$, and hence defines a closed 1-form $\theta$ on $M$. We see easily that $d \beta=\theta \wedge \beta$.

Let $\mathscr{D}_{\alpha_{0}, \text { conf }}(M)$ be the group of all $C^{\infty}$ diffeomorphisms $\varphi$ such that $\varphi^{*} \alpha_{0}=c(\varphi) \alpha_{0}$, where $c(\varphi)$ is a positive constant depending on $\varphi$. Let $\tilde{\varphi}$ be a lift of $\varphi$ onto $\tilde{M}$. Then we have $\tilde{\varphi}^{*} \tilde{\alpha}_{0}=c(\varphi) \tilde{\alpha}_{0}$ and $\psi^{*}\left(\tilde{\varphi}^{*} g / g\right)=\tilde{\varphi}^{*} g / g$ for any $\psi \in \Pi$, because $\psi \tilde{\varphi}=\ddot{\varphi} \psi$. Moreover, $\tilde{\varphi}^{*} \tilde{\beta}=\left(\tilde{\varphi}^{*} g / g\right) c(\varphi) \tilde{\beta}$; hence $\left(\tilde{\varphi}^{*} g / g\right) c(\varphi)$ defines a positive $C^{\infty}$ function $f_{\varphi}$ on $M$ such that $\varphi^{*} \beta=f_{\varphi} \beta$. It is easy to see that $\left(\varphi^{\prime} \varphi\right)^{*} \beta=\left(\varphi^{*} f_{\varphi^{\prime}}\right) \cdot f_{\varphi} \cdot \beta$.

Let $\Gamma\left(1_{M}\right)$ be the space of all $C^{\infty}$ functions on $M$ and $\Gamma_{+}\left(1_{M}\right)$ the space of all positive functions on $M . \Gamma_{+}\left(1_{M}\right)$ is a group under natural multiplications. We make a semidirect product $\Gamma_{+}\left(1_{M}\right) * \mathscr{D}(M)$ (where $\mathscr{D}(M)$ is the group of all $C^{\infty}$ diffeomorphisms of $\left.M\right)$ by defining $\left(f^{\prime}, \varphi^{\prime}\right) *(f, \varphi)=\left(\left(\varphi^{*} f^{\prime}\right) f, \varphi^{\prime} \varphi\right)$.

There is a natural inclusion $i$ of $\mathscr{D}_{\alpha_{0}, \text { conf }}(M)$ into $\Gamma_{+}\left(1_{M}\right) * \mathscr{D}(M)$ given by $i(\varphi)=\left(f_{\varphi}, \varphi\right)$.

Now, as usual, we can define a Lie algebra structure on $\Gamma\left(1_{M}\right) \oplus \mathfrak{X}(M)$ associated with the above group structure. This is given as follows:

$$
\left[(f, u),\left(f^{\prime}, u^{\prime}\right)\right]=\left(u^{\prime} f-u f^{\prime},\left[u, u^{\prime}\right]\right), \quad \text { where }\left[u, u^{\prime}\right]=u^{\prime} u-u u^{\prime} .
$$

Let $\Gamma\left(T_{M}^{*}\right)$ be the space of all $C^{\infty}$ 1-forms on $M$ and $\Gamma\left(\wedge^{k} T_{M}^{*}\right)$ the space of all $C^{\infty} k$-forms on $M$. We define an (anti) action $\rho$ of $\Gamma_{+}\left(1_{M}\right) * \mathscr{D}(M)$ on the space $\Gamma\left(\wedge^{e} T_{M}^{*}\right) \oplus \Gamma\left(T_{M}^{*}\right), \varepsilon=2$ or $n$, by $\rho\left(e^{f}, \varphi\right)(\xi, \eta)=\left(e^{-f} \varphi^{*} \xi, \varphi^{*} \eta-d f\right)$.

If $\left(e^{f}, \varphi\right)(\beta, \theta)=(\beta, \theta)$, then $\varphi^{*} \beta=e^{f} \beta$ and $d f=\varphi^{*} \theta-\theta$. Since $\tilde{\theta}$ $=d g / g$, the second equality shows that $d \tilde{f}=d\left(\log \left(\tilde{\varphi}^{*} g / g\right)\right)$, where $\tilde{f}$ is the pull-back of $f$ onto $\tilde{M}$. Hence, we have $e^{\tilde{f}}=c \tilde{\varphi}^{*} g / g$, where $c$ is a constant. From the first equality, we have $\varphi^{*} \tilde{\alpha}_{0}=c \tilde{\alpha}_{0}$. Thus, the constant $c$ is determined by $\varphi$, and, moreover, $e^{\tilde{f}}=c(\varphi) \tilde{\varphi}^{*} g / g ;$ hence $\left(e^{f}, \varphi\right)$ $\in \mathcal{D}_{\alpha_{0}, \text { conf }}(M)$. The converse is easy to check.

Consider a curve $\left(e^{f_{t}}, \varphi_{t}\right)$ such that $f_{0} \equiv 0, \varphi_{0}=\mathrm{id}$, and $f_{t}, \varphi_{t}$ are of class $C^{\infty}$ with respect to $(t, x), x \in M$. Set $h=\partial /\left.\partial_{t}\right|_{t=0} f_{t}, u=\partial /\left.\partial_{t}\right|_{t=0} \varphi_{t}$. Taking the derivative of $\rho\left(e^{f_{t}}, \varphi_{t}\right)(\beta, \theta)$ at $t=0$, we have the following linear map:

$$
\left.\left.\left.A(h, u)=\left(\mathfrak{R}_{u} \beta-h \beta, \mathfrak{L}_{u} \theta-d h\right)=(d(\beta\lrcorner u)+(d \beta)\right\lrcorner u-h \beta, d(\theta\lrcorner u\right)-d h\right) .
$$

We regard $A$ as a linear mapping of $\Gamma\left(1_{M}\right) \oplus \mathfrak{X}(M)$ into $\Gamma\left(\wedge^{e} T_{M}^{*}\right) \oplus \Gamma\left(T_{M}^{*}\right)$. Now, if $A(h, u)=0$, then $h=\theta\lrcorner u-c$, where $c$ is a constant. Using the first equality $\mathfrak{l}_{u} \beta=h \beta$, we have 


$$
\left.\left.(d g\lrcorner \tilde{u}) \tilde{\alpha}_{0}+g d\left(\tilde{\alpha}_{0}\right\lrcorner \tilde{u}\right)-g\{\tilde{\theta}\lrcorner \tilde{u}-c\right\} \tilde{\alpha}_{0}=0,
$$

where $\tilde{u}$ is the lift of $u$ onto $\tilde{M}$.

We remark that $\tilde{\theta}=d g / g$ and we see $\left.d\left(\tilde{\alpha}_{0}\right\lrcorner \tilde{u}\right)+c \tilde{\alpha}_{0}=0$. Therefore, the constant $c$ is determined by $u$ and, moreover, $\mathfrak{L}_{u} \alpha_{0}=c(u) \alpha_{0}$. We denote by $\mathfrak{X}_{\alpha_{0}, \text { conf }}(M)$ the Lie algebra of all $u \in \mathfrak{X}(M)$ such that $\mathfrak{L}_{u} \alpha_{0}=c(u) \alpha_{0}$. The above argument shows the following:

Lemma 9.1. The Lie algebra $\mathfrak{X}_{\alpha_{0}, \text { conf }}(M)$ is given by the kernel of $A$ : $\Gamma\left(1_{M}\right) \oplus \mathfrak{X}(M) \rightarrow \Gamma\left(\wedge^{\varepsilon} T_{M}^{*}\right) \oplus \Gamma\left(T_{M}^{*}\right), A(h, u)=\left(\mathfrak{L}_{u} \beta-h \beta, \mathfrak{R}_{u} \theta-d h\right)$. The canonical inclusion of $\mathfrak{X}_{\alpha_{0}, \text { conf }}(M)$ into $\Gamma\left(1_{M}\right) \oplus \mathfrak{X}(M)$ is given by $u \mapsto(\theta\lrcorner u$ $-c(u), u)$.

Let $\kappa: 1_{M} \oplus T_{M} \rightarrow \wedge^{\varepsilon-1} T_{M}^{*} \oplus 1_{M}$ be the bundle morphism defined by $\kappa(\lambda, X)=(\beta\lrcorner X, \lambda-\theta\lrcorner X)$, where $1_{M}$ is the trivial bundle $\mathbf{R} \times M$ and $T_{M}, T_{M}^{*}$ are the tangent and the cotangent bundles of $M$, respectively. Since $\beta$ is of maximal rank, it is clear that $\kappa$ is an isomorphism. Define a differential operator $D$ by the following:

$$
\begin{gathered}
D: \Gamma\left(\wedge^{\varepsilon-1} T_{M}^{*}\right) \oplus \Gamma\left(1_{M}\right) \rightarrow \Gamma\left(\wedge^{\varepsilon} T_{M}^{*}\right) \oplus \Gamma\left(T_{M}^{*}\right), \\
D(\eta, g)=(d \eta-\theta \wedge \eta-g \beta,-d g) .
\end{gathered}
$$

Then it is easy to see that $A=D \kappa$. Similarly, we define a differential operator $D$ of $\Gamma\left(\wedge^{e-1+i} T_{M}^{*}\right) \oplus \Gamma\left(\wedge^{i} T_{M}^{*}\right)$ into $\Gamma\left(\wedge^{e+i} T_{M}^{*}\right) \oplus \Gamma\left(\wedge^{i+1} T_{M}^{*}\right)$ by

$$
D(\xi, \eta)=(d \xi-\theta \wedge \xi-\eta \wedge \beta,-d \eta),
$$

where $-\infty<i<\infty$ and $\wedge^{j} T_{M}^{*}=\{0\}$ for $j<0$. We see easily $D^{2}=0$. Let $D^{*}$ be the formal adjoint operator with respect to an arbitrarily fixed riemannian metric on $M$.

Lemma 9.2. $\square=D D^{*}+D^{*} D$ is an elliptic operator.

The proof is trivial, because the symbol $\sigma(D)$ is equal to that of the operator $D^{\prime}(\xi, \eta)=(d \xi,-d \eta)$.

(B) We have now the following diagram:

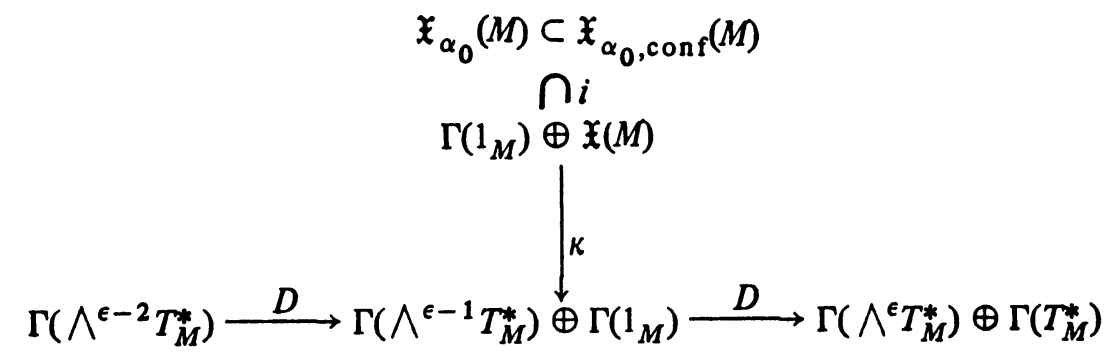

where $\mathfrak{X}_{\alpha_{0}}(M)$ is defined in $\left.\S 8, i(u)=(\theta\lrcorner u-c(u), u\right)$ and $D: \Gamma\left(\wedge^{c-2} T_{M}^{*}\right)$ 
$\rightarrow \Gamma\left(\wedge^{\varepsilon-1} T_{M}^{*}\right) \oplus \Gamma\left(1_{M}\right)$ is given by $D(\xi)=(d \xi-\theta \wedge \xi, 0)$. By definition, $c(u)=0$ for any $u \in \mathfrak{X}_{\alpha_{0}}(M)$. Therefore $\kappa i\left(\mathfrak{X}_{\alpha_{0}}(M)\right) \subset \Gamma\left(\wedge^{\varepsilon-1} T_{M}^{*}\right) \oplus\{0\}$, i.e., $\kappa i(u)=(\beta\lrcorner u, 0)$.

Now, we set

$$
\mathfrak{X}_{\alpha_{0}, c}(M)=\left\{u \in \mathfrak{X}_{\alpha_{0}}(M) ; \kappa i(u)=D \gamma, \operatorname{supp} \gamma \text { is compact }\right\},
$$

where, of course, $\alpha_{0}$ is a multivalued symplectic or volume structure.

LEMMA 9.3. $\mathfrak{X}_{\alpha_{0}, c}(M)$ is the core of $\mathfrak{X}_{\alpha_{0}}(M)$ and of $\mathfrak{X}_{\alpha_{0}, \text { conf }}(M)$.

Proof. It is easy to see using a partition of unity that $\mathfrak{X}_{\alpha_{0}, c}(M)$ has property (P). Therefore it is contained in the cores of $\mathfrak{X}_{\alpha_{0}}(M)$ and $\mathfrak{X}_{\alpha_{0}, \text { conf }}(M)$. To prove

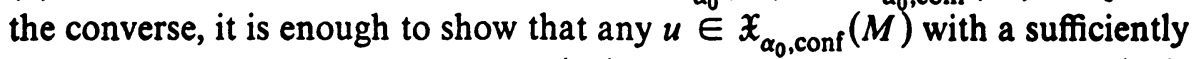
small support is contained in $\mathfrak{X}_{\alpha_{0}, c}(M)$. It is easy to see that $u \in \mathfrak{X}_{\alpha_{0}}(M)$, because $\left.\alpha_{0}\right\lrcorner u \equiv 0$ outside of the support.

Assume that supp $u$ is contained in an evenly covered open ball $U$ in $M$. Let $\pi: \tilde{M} \rightarrow M$ be the projection. $\tilde{U}=\pi^{-1}(U)$ is then a disjoint union of open balls $\psi\left(U^{\prime}\right), \psi \in \Pi$, where $\Pi$ is the covering transformation group and $U^{\prime}$ is an open ball in $\tilde{M}$ which is diffeomorphic to $U$ through the projection $\pi$. Now, recall that $\kappa i(u)=(\beta\lrcorner u, 0)$ and $d(\beta\lrcorner u)-\theta \wedge(\beta\lrcorner u)=0$. Therefore, $d(\tilde{\beta}\lrcorner \tilde{u})$ $\left.-\tilde{\theta} \wedge(\tilde{\beta}\lrcorner \tilde{u})=g d\left(\tilde{\alpha}_{0}\right\lrcorner \tilde{u}\right)=0$, where $\tilde{u}$ is the lift of $u$ onto $\tilde{M}$. By the Poincaré lemma, there is $\gamma^{\prime}$ such that $\left.\left(\tilde{\alpha}_{0}\right\lrcorner \tilde{u}\right) \mid U^{\prime}=d \gamma^{\prime}$ and $\operatorname{supp} \gamma^{\prime} \subset U^{\prime}$. Let $\tau(\psi)$ be the constant defined by $\psi^{*} \tilde{\alpha}_{0}=\tau(\psi) \tilde{\alpha}_{0}$. Then we have

$$
\left.\tilde{\alpha}_{0}\right\lrcorner \tilde{u}=d \sum_{\psi \in \Pi} \tau(\psi)^{-1} \psi^{*} \gamma^{\prime}
$$

Since $\psi U^{\prime} \cap \psi^{\prime} U^{\prime}=\varnothing$ for any $\psi, \psi^{\prime} \in \Pi$ such that $\psi \neq \psi^{\prime}$, the right-hand side is well defined. Note that $\psi^{*} g=\tau(\psi)^{-1} g$. We have then

$$
\tilde{\beta}\lrcorner \tilde{u}=d\left(\sum_{\psi \in \Pi} \psi^{*}\left(g \gamma^{\prime}\right)\right)-\tilde{\theta} \wedge\left(\sum_{\psi \in \Pi} \psi^{*}\left(g \gamma^{\prime}\right)\right) .
$$

Since $\sum_{\psi \in \Pi} \psi^{*}\left(g \gamma^{\prime}\right)$ is invariant under the action of $\Pi$, this defines a form $\gamma$ on $M$ such that $\operatorname{supp} \gamma \subset U$, and $\beta\lrcorner u=d \gamma-\theta \wedge \gamma=D \gamma$. This proves the lemma.

\section{REFERENCES}

1. R. Abraham, Foundations of mechanics, Benjamin, New York, 1967. MR 36 \#3527.

2. I. Amemiya, Lie algebra of vector fields and complex structure, J. Math. Soc. Japan 27 (1975), 545-549.

3. V. W. Guillemin, Infinite dimensional primitive Lie algebras, J. Differential Geometry 4 (1970), 257-282. MR $42 \# 3132$.

4. A. Koriyama, On Lie algebras of vector fields with invariant submanifolds, Nagoya Math. J. 55 (1974), 91-110. 
5. B. Malgrange, Ideals of differentiable functions, Tata Inst. of Fundamental Research, Bombay; Oxford Univ. Press, London, 1967. MR 35 \#3446.

6. S. Mizohata, The theory of partial differential equations, Comtemporary Math., no. 9, Iwanami, Shoten, Tokyo, 1965. MR 38 \# 396.

7. T. Morimoto and N. Tanaka, The classification of real primitive infinite Lie algebras, J. Math. Kyoto Univ. 10 (1970), 207-243. MR 42 \#3133.

8. T. Ochiai, On the automorphism group of a G-structure, J. Math. Soc. Japan 18 (1966), 189-193. MR 33 \#3224.

9. H. Omori, Infinite dimensional Lie transformation groups, Lecture Notes in Math., vol. 427, Springer-Verlag, Berlin and New York, 1974.

10. L. E. Pursell and M. E. Shanks, The Lie algebra of a smooth manifold, Proc. Amer. Math. Soc. 5 (1954), 468-472. MR 16, 331.

11. S. Shnider, The classification of real primitive infintie Lie algebras, J. Differential Geometry 4 (1970), 81-89. MR 44 \#2792.

12. I. M. Singer and S. Sternberg, The infinite groups of Lie and Cartan. I, J. Analyse Math. 15 (1965), 1-114. MR 36 \#911.

13. H. J. Sussmann, Orbits of families of vector fields and integrability of distributions, Trans. Amer. Math. Soc. 180 (1973), 171-188.

Department of Mathematical Sciences, Tokaj University, Hiratsuka, Kanagawa, Japan (Current address of A. Koriyama)

Department of Mathematics, Tokyo Metropolttan University, Setagaya, Tokyo, Japan (Current address of Y. Maeda and H. Omori) 